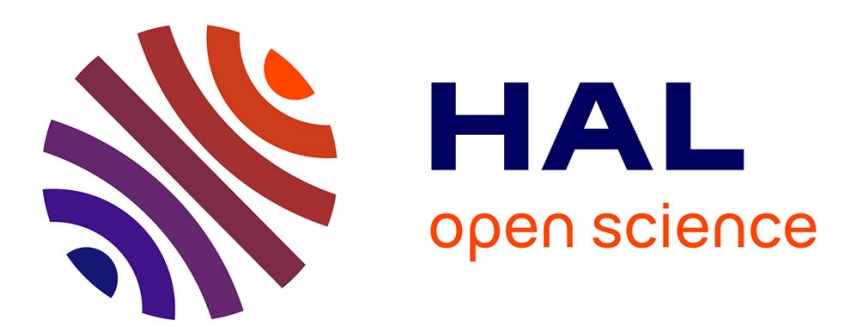

\title{
Non-parametric estimation of extreme risk measures from conditional heavy-tailed distributions
}

\author{
Jonathan El Methni, Laurent Gardes, Stéphane Girard
}

\section{To cite this version:}

Jonathan El Methni, Laurent Gardes, Stéphane Girard. Non-parametric estimation of extreme risk measures from conditional heavy-tailed distributions. Scandinavian Journal of Statistics, 2014, 41 (4), pp.988-1012. 10.1111/sjos.12078 . hal-00830647v5

\section{HAL Id: hal-00830647 \\ https://hal.science/hal-00830647v5}

Submitted on 28 Nov 2013

HAL is a multi-disciplinary open access archive for the deposit and dissemination of scientific research documents, whether they are published or not. The documents may come from teaching and research institutions in France or abroad, or from public or private research centers.
L'archive ouverte pluridisciplinaire HAL, est destinée au dépôt et à la diffusion de documents scientifiques de niveau recherche, publiés ou non, émanant des établissements d'enseignement et de recherche français ou étrangers, des laboratoires publics ou privés. 


\title{
Nonparametric estimation of extreme risk measures from conditional heavy-tailed distributions
}

\author{
Jonathan El Methni $^{(1)}$, Laurent Gardes ${ }^{(2)} \&$ Stéphane Girard $^{(3)}$
}

(1) Université de Genève, 40 boulevard du Pont-d'Arve, 1205 Genève, Switzerland.

(2) Université de Strasbourg \& CNRS, IRMA, UMR 7501, 7 rue René Descartes, 67084 Strasbourg cedex, France.

(3) Team Mistis, INRIA Rhône-Alpes \& LJK, Inovallée, 655, av. de l'Europe, Montbonnot, 38334 Saint-Ismier cedex, France.

\begin{abstract}
In this paper, we introduce a new risk measure, the so-called Conditional Tail Moment. It is defined as the moment of order $a \geq 0$ of the loss distribution above the upper $\alpha$-quantile where $\alpha \in(0,1)$. Estimating the Conditional Tail Moment permits to estimate all risk measures based on conditional moments such as Conditional Tail Expectation, Conditional Value-atRisk or Conditional Tail Variance. Here, we focus on the estimation of these risk measures in case of extreme losses (where $\alpha \rightarrow 0$ is no longer fixed). It is moreover assumed that the loss distribution is heavy-tailed and depends on a covariate. The estimation method thus combines nonparametric kernel methods with extreme-value statistics. The asymptotic distribution of the estimators is established and their finite sample behavior is illustrated both on simulated data and on a real data set of daily rainfalls.
\end{abstract}

Keywords: Conditional tail Expectation, Heavy-tailed distributions, Kernel estimator, Asymptotic normality, Risk measures, Extreme-value statistics.

AMS 2000 subject classification: 62G32, 62G30, 62E20.

\section{Introduction}

One of the most popular risk measures is the Value-at-Risk (VaR) introduced in the 1990's, see [22] for a review. In statistical terms, the VaR at level $\alpha \in(0,1)$ corresponds to the upper $\alpha$-quantile of the loss distribution. The Value-at-Risk however suffers from several weaknesses. First, it provides us only with a pointwise information: $\operatorname{VaR}(\alpha)$ does not take into consideration what the loss will be beyond this quantile. Second, random loss variables with light-tailed distributions or heavy-tailed distributions may have the same Value-at-Risk [31]. Finally, Value-at-Risk is not a coherent risk measure [1] since it is not subadditive in general ${ }^{1}$.

A coherent alternative risk measure is the Conditional Tail Expectation (CTE) [1], also known as Tail-Value-at-Risk, Tail Conditional Expectation or Expected Shortfall in case of a continuous

\footnotetext{
${ }^{1}$ Recall that a risk measure $\rho$ is subadditive if $\rho(Z+T) \leq \rho(Z)+\rho(T)$ for all random loss variables $Z$ and $T$.
} 
loss distribution. The CTE is defined as the expected loss given that the loss lies above the upper $\alpha$-quantile of the loss distribution. This risk measure thus takes into account the whole information contained in the upper tail of the distribution. It has been extensively studied in $[1,5,31]$ and is frequently encountered in financial investment or in the insurance industry [4, 23].

Other existing risk measures include: the Conditional Tail Variance (CTV) [32], the Conditional Tail Skewness (CTS) [20] respectively defined as the variance or skewness of the loss distribution above the upper $\alpha$-quantile, the Conditional-Value-at-Risk (CVaR) [29] defined as the weighted average between VaR and CTE, and the Stop-loss Premium reinsurance risk measure (SP) with retention level equal to VaR [5], see Section 2 for a precise definition.

In this paper, we first introduce a new tool for unifying the estimation of the above mentioned risk measures: the Conditional Tail Moment (CTM). It is defined as the moment of order $a \geq 0$ of the random loss distribution above the VaR at level $\alpha$. We shall show that estimating the CTM permits to estimate all risk measures based on conditional moments of arbitrary orders above the VaR. For instance, it is clear that the Conditional Tail Moment of order one reduces to the Conditional Tail Expectation. Our second contribution is to investigate the estimation of the CTM in case of extreme losses $(\alpha \rightarrow 0)$ making heavy use of the extreme-value theory. Even though links between extreme-value theory and risk measures have already been investigated [11, 12, 24], the estimation of risk measures is usually achieved in the statistical literature for fixed values of $\alpha$, see for instance $[8,25,26]$. Our third contribution is to propose an estimator of extreme risk measures able to deal with covariates. In this context, the new risk measure is referred to as the Regression Conditional Tail Moment (RCTM). For instance, in finance, the loss distribution can be affected by many factors, such as interest rates or inflation. In meteorology, one is interested in the extreme rainfalls as a function of the geographical location $[9,14]$.

The paper is organized as follows. The definition of the RCTM and its link with classical risk measures are given in Section 2. Asymptotic properties are established in Section 3. The efficiency of our estimators is then illustrated on simulated and real data in Section 4. Proofs are postponed to the Appendix.

\section{The Regression Conditional Tail Moment: definition and estimation}

\subsection{A new risk measure}

Let $Y \in \mathbb{R}$ be a random loss variable. For $\alpha \in(0,1)$, the Value at Risk of level $\alpha$ is the quantity $\operatorname{VaR}(\alpha)$ satisfying $\mathbb{P}(Y>\operatorname{VaR}(\alpha))=\alpha$. The Value at Risk is the most popular risk measure [22] but many others can be found in the literature:

- The Conditional Tail Expectation [1] is defined by $\operatorname{CTE}(\alpha):=\mathbb{E}(Y \mid Y>\operatorname{VaR}(\alpha))$.

- The Conditional Tail Variance $\operatorname{CTV}(\alpha):=\mathbb{E}\left((Y-\operatorname{CTE}(\alpha))^{2} \mid Y>\operatorname{VaR}(\alpha)\right)$ was introduced in [32]. It measures the conditional variability of $Y$ given $Y>\operatorname{VaR}(\alpha)$ and indicates how far away the events deviate from $\operatorname{CTE}(\alpha)$.

- The Conditional Tail Skewness $\operatorname{CTS}(\alpha):=\mathbb{E}\left(Y^{3} \mid Y>q(\alpha)\right) /(\operatorname{CTV}(\alpha))^{3 / 2}$ was defined in [20].

- The Conditional-Value-at-Risk is defined by $\operatorname{CVaR}_{\lambda}(\alpha):=\lambda \operatorname{VaR}(\alpha)+(1-\lambda) \operatorname{CTE}(\alpha)$ with $0 \leq$ $\lambda \leq 1$. It is clear that $\operatorname{CVaR}_{1}(\alpha)=\operatorname{VaR}(\alpha)$ and $\operatorname{CVaR}_{0}(\alpha)=\operatorname{CTE}(\alpha)$. This risk measure is 
able to quantify dangers beyond $\operatorname{VaR}(\alpha)$ and is moreover coherent for $\lambda \neq 1$. Other fundamental properties can be found in [29].

- The Stop-loss Premium reinsurance risk measure with retention level equal to $\operatorname{VaR}(\alpha)[5]$ is proportional to the difference between $\operatorname{CTE}(\alpha)$ and $\operatorname{VaR}(\alpha): \operatorname{SP}(\alpha):=\mathbb{E}\left((Y-\operatorname{VaR}(\alpha))_{+}\right)=$ $\alpha(\operatorname{CTE}(\alpha)-\operatorname{VaR}(\alpha))$, where $z_{+}=\max (0, z)$. This measure thus permits to emphasize the dangerous cases.

The first purpose of this paper is to unify the definitions of the above risk measures. To this end, a new risk measure is introduced, the Conditional Tail Moment $\mathrm{CTM}_{a}(\alpha):=\mathbb{E}\left(Y^{a} \mid Y>\operatorname{VaR}(\alpha)\right)$, where $a \geq 0$ is such that the moment of order $a$ of $Y$ exists. It is easy to check that all the above risk measures of level $\alpha$ can be rewritten as $\Phi\left(\operatorname{VaR}(\alpha), \mathrm{CTM}_{1}(\alpha), \mathrm{CTM}_{2}(\alpha), \mathrm{CTM}_{3}(\alpha)\right)$, where the function $\Phi: \mathbb{R}^{4} \mapsto \mathbb{R}$ is taken in Table 1 .

\begin{tabular}{|c|c|}
\hline Risk measure & $\Phi\left(t_{0}, t_{1}, t_{2}, t_{3}\right)$ \\
\hline $\operatorname{CTE}(\alpha)$ & $t_{1}$ \\
$\operatorname{CTV}(\alpha)$ & $t_{2}-t_{1}^{2}$ \\
$\operatorname{CTS}(\alpha)$ & $t_{3} /\left(t_{2}-t_{1}^{2}\right)^{3 / 2}$ \\
$\operatorname{CVaR}(\alpha)$ & $\lambda t_{0}+(1-\lambda) t_{1}, \lambda \in[0,1]$ \\
$\operatorname{SP}(\alpha)$ & $\alpha\left(t_{1}-t_{0}\right)$ \\
\hline
\end{tabular}

Table 1: Links between the new risk measure and classical risk measures

More generally, the CTM can be used to define any risk measure based on conditional moments of the loss variable above the VaR of level $\alpha$. For instance, one could introduce the conditional tail kurtosis thanks via the function $\Phi\left(t_{0}, t_{1}, t_{2}, t_{3}, t_{4}\right)=t_{4} /\left(t_{2}-t_{1}^{2}\right)^{2}$.

\subsection{Extreme losses and regression case}

As announced in the introduction, our second purpose is to adapt the classical risk measures to extreme losses and to the case where a covariate $X \in \mathbb{R}^{p}$ is recorded simultaneously with the loss variable $Y$. To this end, the fixed level $\alpha \in(0,1)$ is replaced by a sequence $\left(\alpha_{n}\right) \in(0,1)$, such that $\alpha_{n} \rightarrow 0$. Furthermore, denoting by $\bar{F}(. \mid x)$ the conditional survival distribution function of $Y$ given $X=x$, we define the Regression Value-at Risk by $\operatorname{RVaR}\left(\alpha_{n} \mid x\right):=\bar{F}^{\leftarrow}\left(\alpha_{n} \mid x\right)=\inf \left\{t, \bar{F}(t \mid x) \leq \alpha_{n}\right\}$, and the Regression Conditional Tail Moment of order $a$ by:

$$
\operatorname{RCTM}_{a}\left(\alpha_{n} \mid x\right):=\mathbb{E}\left(Y^{a} \mid Y>\operatorname{RVaR}\left(\alpha_{n} \mid x\right), X=x\right)
$$

where $a>0$ is such that the moment of order $a$ of $Y$ exists. Note that in this framework, $\operatorname{RVaR}\left(\alpha_{n} \mid x\right)$ is the extreme conditional quantile of level $\alpha_{n} \in(0,1)$, see for instance $[2,9,10,14$, $15,34]$. It is then quite easy to adapt the classical risk measures to extreme losses and to the presence of a covariate by applying the desired function (see Table 1) to the vector

$$
\left(\operatorname{RVaR}\left(\alpha_{n} \mid x\right), \operatorname{RCTM}_{1}\left(\alpha_{n} \mid x\right), \operatorname{RCTM}_{2}\left(\alpha_{n} \mid x\right), \operatorname{RCTM}_{3}\left(\alpha_{n} \mid x\right)\right) .
$$

This yields the following risk measures: $\operatorname{RCTE}\left(\alpha_{n} \mid x\right), \operatorname{RCTV}\left(\alpha_{n} \mid x\right), \operatorname{RCTS}\left(\alpha_{n} \mid x\right), \operatorname{RCVaR}\left(\alpha_{n} \mid x\right)$ and $\operatorname{RSP}\left(\alpha_{n} \mid x\right)$. 


\section{$2.3 \quad$ Inference}

Let $\left(X_{i}, Y_{i}\right), i=1, \ldots, n$, be independent copies of the random pair $(X, Y)$. To estimate the $\mathrm{RCTM}$, we start from the following straightforward equality

$$
\operatorname{RCTM}_{a}\left(\alpha_{n} \mid x\right)=\frac{1}{\alpha_{n}} \varphi_{a}\left(\varphi_{0}^{\leftarrow}\left(\alpha_{n} \mid x\right) \mid x\right)
$$

where for $y>0, \varphi_{a}(y \mid x)=\mathbb{E}\left(Y^{a} \mathbb{I}\{Y>y\} \mid X=x\right)$ is the conditional moment of order $a \geq 0$. Estimation of the RCTM thus relies on the estimation of the conditional moment. We propose to use a classical kernel estimator (see $[27,30]$ ) given by

$$
\hat{\varphi}_{a, n}(y \mid x)=\sum_{i=1}^{n} K_{h}\left(x-X_{i}\right) Y_{i}^{a} \mathbb{I}\left\{Y_{i}>y\right\} / \sum_{i=1}^{n} K_{h}\left(x-X_{i}\right),
$$

where $\mathbb{I}\{$.$\} is the indicator function and h=h_{n}$ is a non-random sequence such that $h \rightarrow 0$ as $n \rightarrow \infty$. We have also introduced $K_{h}(t)=K(t / h) / h^{p}$ where $K$ is a density on $\mathbb{R}^{p}$. In this context, $h$ is called the window-width. Since $\hat{\varphi}_{a, n}(. \mid x)$ is a non increasing function, we can define an estimator of $\varphi_{a}^{\leftarrow}(\alpha \mid x)$ for $\alpha \in(0,1)$ by

$$
\hat{\varphi}_{a, n}^{\leftarrow}(\alpha \mid x)=\inf \left\{t, \hat{\varphi}_{a, n}(t \mid x)<\alpha\right\} .
$$

Remarking that $\varphi_{0}(y \mid x)=\bar{F}(y \mid x)$, the RVaR of level $\alpha_{n}$ is thus estimated by

$$
\widehat{\operatorname{RVaR}}_{n}\left(\alpha_{n} \mid x\right)=\hat{\varphi}_{0, n}^{\leftarrow}\left(\alpha_{n} \mid x\right)
$$

We thus recover the extreme conditional quantile estimator studied in $[9,10]$. The RCTM of order $a$ is estimated by

$$
\widehat{\operatorname{RCTM}}_{a, n}\left(\alpha_{n} \mid x\right)=\frac{1}{\alpha_{n}} \hat{\varphi}_{a, n}\left(\hat{\varphi}_{0, n}^{\leftarrow}\left(\alpha_{n} \mid x\right) \mid x\right)
$$

An estimator of each of the above mentioned risk measures is thus given by

$$
\Phi\left(\widehat{\operatorname{RVaR}}_{n}\left(\alpha_{n} \mid x\right), \widehat{\operatorname{RCTM}}_{1, n}\left(\alpha_{n} \mid x\right), \widehat{\operatorname{RCTM}}_{2, n}\left(\alpha_{n} \mid x\right), \widehat{\operatorname{RCTM}}_{3, n}\left(\alpha_{n} \mid x\right)\right),
$$

where the function $\Phi$ is chosen in Table 1. The obtained estimators will be denoted by $\widehat{\operatorname{RCTE}}_{n}\left(\alpha_{n} \mid x\right)$, $\widehat{\operatorname{RCTV}}_{n}\left(\alpha_{n} \mid x\right), \widehat{\operatorname{RCTS}}_{n}\left(\alpha_{n} \mid x\right), \widehat{\mathrm{RCVaR}}_{\lambda, n}\left(\alpha_{n} \mid x\right)$ and $\widehat{\mathrm{RSP}}_{n}\left(\alpha_{n} \mid x\right)$. As an example, the estimated RCTE is simply given by $\widehat{\operatorname{RCTE}}_{n}\left(\alpha_{n} \mid x\right)=\hat{\varphi}_{1, n}\left(\hat{\varphi}_{0, n}^{\leftarrow}\left(\alpha_{n} \mid x\right) \mid x\right) / \alpha_{n}$. The joint asymptotic distribution of the RCTM and RVaR estimators, and consequently of all the above mentioned estimators, is established in the next section.

\section{Main results}

Our main assumption is the following:

(F.1) We assume that the conditional survival distribution function of $Y$ given $X=x$ is heavytailed and admits a probability density function.

To summarize, (F.1) amounts to assuming that the conditional distribution of $Y$ given $X=x$ is in the Fréchet maximum domain of attraction. Assumption (F.1) is also equivalent to stating 
that for all $y>0, \bar{F}(y \mid x)=\mathbb{P}(Y>y \mid X=x)$ is regularly varying at infinity (see [3]) with index $-1 / \gamma(x)$ denoted by $\bar{F}(. \mid x) \in \mathcal{R} \mathcal{V}_{-1 / \gamma(x)}$ i.e for all $\lambda>0$,

$$
\lim _{y \rightarrow \infty} \frac{\bar{F}(\lambda y \mid x)}{\bar{F}(y \mid x)}=\lambda^{-1 / \gamma(x)} .
$$

In this context, $\gamma($.$) is a positive function of the covariate x$ and is referred to as the conditional tail index since it tunes the tail heaviness of the conditional distribution of $Y$ given $X=x$. It also appears that, under (F.1), a sufficient condition for the existence of $\operatorname{RCTM}_{a}(1 / . \mid x)$ is $a<1 / \gamma(x)$. As established in Lemma 1, condition (F.1) also implies that, for all $a \in[0,1 / \gamma(x)$ ), $\varphi_{a}(. \mid x) \in \mathcal{R}_{a-1 / \gamma(x)}$. Since, moreover, $\bar{F}(. \mid x) \in \mathcal{R} \mathcal{V}_{-1 / \gamma(x)}$, we have $\operatorname{RCTM}_{a}(1 / . \mid x) \in \mathcal{R} \mathcal{V}_{a \gamma(x)}$. This is equivalent to state that for $a \in[0,1 / \gamma(x))$ and for all $y>0$,

$$
\operatorname{RCTM}_{a}(1 / y \mid x)=y^{a \gamma(x)} \ell_{a}(y \mid x),
$$

with a positive index $a \gamma(x)$ and, for $x$ fixed, $\ell_{a}(. \mid x)$ is a slowly-varying function at infinity, i.e for all $\lambda>0$,

$$
\lim _{y \rightarrow \infty} \frac{\ell_{a}(\lambda y \mid x)}{\ell_{a}(y \mid x)}=1
$$

To establish the asymptotic normality of (4), the following additional conditions are required. First, as remarked in [3], p.15, since slowly-varying functions are of interest only asymptotically, one can assume without loosing generality that in (6)

(F.2) $\ell_{a}(. \mid x)$ is normalized for all $a \in[0,1 / \gamma(x))$.

In such a case, the Karamata representation (see [3], Theorem 1.3.1) of the slowly-varying function can be written as

$$
\ell_{a}(y \mid x)=c_{a}(x) \exp \left(\int_{1}^{y} \frac{\varepsilon_{a}(u \mid x)}{u} d u\right)
$$

where $c_{a}($.$) is a positive function and \varepsilon_{a}(y \mid x) \rightarrow 0$ as $y \rightarrow \infty$. Thus, $\ell_{a}(. \mid x)$ is differentiable and the auxiliary function is given by $\varepsilon_{a}(y \mid x)=y \ell_{a}^{\prime}(y \mid x) / \ell_{a}(y \mid x)$. This function plays an important role in extreme-value theory since it drives the speed of convergence in (7) and more generally the bias of extreme-value estimators. Therefore, it may be of interest to specify how it converges to 0 . In [18], the auxiliary function is supposed to be regularly varying and the estimation of the conditional regular variation index is addressed. Here, we limit ourselves to assuming that for all $a \in(0,1 / \gamma(x))$,

(F.3) $\left|\varepsilon_{a}(. \mid x)\right|$ is continuous and ultimately non-increasing.

A Lipschitz condition on the probability density function $g$ of $X$ is also required. For all $\left(x, x^{\prime}\right) \in$ $\mathbb{R}^{p} \times \mathbb{R}^{p}$, the distance between $x$ and $x^{\prime}$ is denoted by $d\left(x, x^{\prime}\right)$ and the following assumption is introduced:

(L) There exists a constant $c_{g}>0$ such that $\left|g(x)-g\left(x^{\prime}\right)\right| \leq c_{g} d\left(x, x^{\prime}\right)$.

The next assumption is standard in the kernel estimation framework.

(K) $K$ is a bounded density on $\mathbb{R}^{p}$, with support $S$ included in the unit ball of $\mathbb{R}^{p}$. 
For $\xi>0$, the largest oscillation at point $(x, y) \in \mathbb{R}^{p} \times \mathbb{R}_{*}^{+}$of the conditional moment of order $a \in[0,1 / \gamma(x))$ is given by

$$
\omega(x, y, a, \xi, h)=\sup \left\{\left|\frac{\varphi_{a}(z \mid x)}{\varphi_{a}\left(z \mid x^{\prime}\right)}-1\right|, z \in[(1-\xi) y,(1+\xi) y] \text { and } x^{\prime} \in B(x, h)\right\},
$$

where $B(x, h)$ denotes the ball centred at $x$ with radius $h$. Finally, for all finite set $E$, let $\mathcal{L}(E)=$ $\left\{e_{i}+e_{j},\left(e_{i}, e_{j}\right) \in E \times E\right\} \cup E$. We are now in position to establish our main result.

Theorem 1 Suppose (F.1), (F.2), (L) and (K) hold. Let us introduce $0 \leq a_{1}<a_{2}<\cdots<a_{J}$ where $J$ is a positive integer. For all $x \in \mathbb{R}^{p}$ such that $g(x)>0$ and $\gamma(x)<1 /\left(2 a_{J}\right)$, let us introduce a sequence $\left(\alpha_{n}\right)$ with $\alpha_{n} \rightarrow 0$ and $n h^{p} \alpha_{n} \rightarrow \infty$ as $n \rightarrow \infty$. If there exists $\xi>0$ such that

$$
n h^{p} \alpha_{n}\left(h \vee \max _{a \in \mathcal{L}\left(\left\{0, a_{1}, \ldots, a_{J}\right\}\right)} \omega\left(x, \varphi_{0}^{\leftarrow}\left(\alpha_{n} \mid x\right), a, \xi, h\right)\right)^{2} \rightarrow 0,
$$

then, the random vector

$$
\sqrt{n h^{p} \alpha_{n}}\left\{\left(\frac{\widehat{\operatorname{RCTM}}_{a_{j}, n}\left(\alpha_{n} \mid x\right)}{\operatorname{RCTM}_{a_{j}}\left(\alpha_{n} \mid x\right)}-1\right)_{j \in\{1, \ldots, J\}},\left(\frac{\widehat{\operatorname{RVaR}}_{n}\left(\alpha_{n} \mid x\right)}{\operatorname{RVaR}\left(\alpha_{n} \mid x\right)}-1\right)\right\}
$$

is asymptotically Gaussian, centred, with a $(J+1) \times(J+1)$ covariance matrix $\|K\|_{2}^{2} \gamma^{2}(x) \Sigma(x) / g(x)$ where for $(i, j) \in\{1, \ldots, J\}^{2}$ we have $\Sigma_{J+1, j}(x)=a_{j}, \Sigma_{i, J+1}(x)=a_{i}, \Sigma_{J+1, J+1}(x)=1$ and

$$
\Sigma_{i, j}(x)=\frac{a_{i} a_{j}\left(2-\left(a_{i}+a_{j}\right) \gamma(x)\right)}{\left(1-\left(a_{i}+a_{j}\right) \gamma(x)\right)} .
$$

Theorem 1 permits to establish the asymptotic normality for any regression estimator of a risk measure based on arbitrary moments above an extreme conditional quantile. In particular, $\widehat{\operatorname{RCTE}}_{n}\left(\alpha_{n} \mid x\right)$, $\widehat{\mathrm{RCVaR}}_{\lambda, n}\left(\alpha_{n} \mid x\right)$ and $\widehat{\mathrm{RSP}}_{n}\left(\alpha_{n} \mid x\right)$ only involve the first order moment, their asymptotic normality can be derived under the assumption $\gamma(x)<1 / 2$ :

Corollary 1 Suppose (F.1), (F.2), (L) and (K) hold. For all $x \in \mathbb{R}^{p}$ such that $g(x)>0$ and $\gamma(x)<1 / 2$, let us introduce a sequence $\left(\alpha_{n}\right)$ with $\alpha_{n} \rightarrow 0$ and $n h^{p} \alpha_{n} \rightarrow \infty$ as $n \rightarrow \infty$. If there exists $\xi>0$ such that

$$
n h^{p} \alpha_{n}\left(h \vee \max _{a \in\{0,1,2\}} \omega\left(x, \varphi_{0}^{\leftarrow}\left(\alpha_{n} \mid x\right), a, \xi, h\right)\right)^{2} \rightarrow 0,
$$

then

$$
\begin{aligned}
\sqrt{n h^{p} \alpha_{n}}\left(\frac{\widehat{\operatorname{RCTE}}_{n}\left(\alpha_{n} \mid x\right)}{\operatorname{RCTE}\left(\alpha_{n} \mid x\right)}-1\right) & \stackrel{d}{\longrightarrow} \mathcal{N}\left(0, \frac{2 \gamma^{2}(x)(1-\gamma(x))}{1-2 \gamma(x)} \frac{\|K\|_{2}^{2}}{g(x)}\right), \\
\sqrt{n h^{p} \alpha_{n}}\left(\frac{\widehat{\operatorname{RCVa}}_{\lambda, n}\left(\alpha_{n} \mid x\right)}{\operatorname{RCVaR}\left(\alpha_{n} \mid x\right)}-1\right) & \stackrel{d}{\longrightarrow} \mathcal{N}\left(0, \frac{\gamma^{2}(x)\left(\lambda^{2}+2-2 \lambda-2 \gamma(x)\right)}{1-2 \gamma(x)} \frac{\|K\|_{2}^{2}}{g(x)}\right), \\
\sqrt{n h^{p} \alpha_{n}}\left(\frac{\widehat{\operatorname{RSP}}_{n}\left(\alpha_{n} \mid x\right)}{\operatorname{RSP}\left(\alpha_{n} \mid x\right)}-1\right) & \stackrel{d}{\longrightarrow} \mathcal{N}\left(0, \frac{\gamma^{2}(x)}{1-2 \gamma(x)} \frac{\|K\|_{2}^{2}}{g(x)}\right) .
\end{aligned}
$$

The $\operatorname{RCTV}\left(\alpha_{n} \mid x\right)$ estimator involves the computation of a second order moment, its asymptotic normality requires the stronger condition $\gamma(x)<1 / 4$. 
Corollary 2 Suppose (F.1), (F.2), (L) and (K) hold. For all $x \in \mathbb{R}^{p}$ such that $g(x)>0$ and $\gamma(x)<1 / 4$, let us introduce a sequence $\left(\alpha_{n}\right)$ with $\alpha_{n} \rightarrow 0$ and $n h^{p} \alpha_{n} \rightarrow \infty$ as $n \rightarrow \infty$. If there exists $\xi>0$ such that

$$
n h^{p} \alpha_{n}\left(h \vee \max _{a \in\{0, \ldots, 4\}} \omega\left(x, \varphi_{0}^{\leftarrow}\left(\alpha_{n} \mid x\right), a, \xi, h\right)\right)^{2} \rightarrow 0
$$

then

$$
\sqrt{n h^{p} \alpha_{n}}\left(\frac{\widehat{\operatorname{RCTV}}_{n}\left(\alpha_{n} \mid x\right)}{\operatorname{RCTV}\left(\alpha_{n} \mid x\right)}-1\right) \stackrel{d}{\longrightarrow} \mathcal{N}\left(0, \frac{8(1-\gamma(x))(1-2 \gamma(x))\left(1+2 \gamma(x)+3 \gamma^{2}(x)\right)}{(1-3 \gamma(x))(1-4 \gamma(x))} \frac{\|K\|_{2}^{2}}{g(x)}\right) .
$$

Similarly, the $\operatorname{RCTS}\left(\alpha_{n} \mid x\right)$ estimator involves the computation of a third order moment, its asymptotic normality requires the even stronger condition $\gamma(x)<1 / 6$.

Corollary 3 Suppose (F.1), (F.2), (L) and (K) hold. For all $x \in \mathbb{R}^{p}$ such that $g(x)>0$ and $\gamma(x)<1 / 6$, let us introduce a sequence $\left(\alpha_{n}\right)$ with $\alpha_{n} \rightarrow 0$ and $n h^{p} \alpha_{n} \rightarrow \infty$ as $n \rightarrow \infty$. If there exists $\xi>0$ such that

$$
n h^{p} \alpha_{n}\left(h \vee \max _{a \in\{0, \ldots, 6\}} \omega\left(x, \varphi_{0}^{\leftarrow}\left(\alpha_{n} \mid x\right), a, \xi, h\right)\right)^{2} \rightarrow 0
$$

then

$$
\sqrt{n h^{p} \alpha_{n}}\left(\frac{\widehat{\operatorname{RCTS}}_{n}\left(\alpha_{n} \mid x\right)}{\operatorname{RCTS}\left(\alpha_{n} \mid x\right)}-1\right) \stackrel{d}{\longrightarrow} \mathcal{N}\left(0, V(x) \frac{\|K\|_{2}^{2}}{g(x)}\right)
$$

where

$$
V(x)=\frac{18\left(1-13 \gamma(x)+50 \gamma^{2}(x)-44 \gamma^{3}(x)-23 \gamma^{4}(x)-3 \gamma^{5}(x)\right)}{(1-3 \gamma(x))(1-4 \gamma(x))(1-5 \gamma(x))(1-6 \gamma(x))} .
$$

In Theorem 1, the condition $n h^{p} \alpha_{n} \rightarrow 0$ provides a lower bound on the level of the risk measure to estimate. This restriction is a consequence of the use of kernel estimator (2) which cannot extrapolate beyond the maximum observation in the ball $B(x, h)$. In consequence, $\alpha_{n}$ must be an order of an extreme quantile within the sample. To overcome this limitation, we propose to adapt Weissman's estimator [35], initially designed for the estimation of unconditional quantiles, to the estimation of the RCTM:

$$
\widehat{\operatorname{RCTM}}_{a, n}^{W}\left(\beta_{n} \mid x\right)=\widehat{\operatorname{RCTM}}_{a, n}\left(\alpha_{n} \mid x\right)\left(\frac{\alpha_{n}}{\beta_{n}}\right)^{a \hat{\gamma}_{n}(x)},
$$

where $a$ is a fixed value, $0<\beta_{n}<\alpha_{n}$ and $\hat{\gamma}_{n}(x)$ is an estimator of the conditional tail-index $\gamma(x)$ (see $[13,16,17,18,33])$. As illustrated in the next theorem, the extrapolation factor $\left(\alpha_{n} / \beta_{n}\right)^{a \hat{\gamma}_{n}(x)}$ allows us to estimate RCTM of arbitrary small levels $\beta_{n}$.

Theorem 2 Suppose the assumptions of Theorem 1 hold together with (F.3). Let us consider $\hat{\gamma}_{n}(x)$ an estimator of the tail index such that

$$
\sqrt{n h_{n}^{p} \alpha_{n}}\left(\hat{\gamma}_{n}(x)-\gamma(x)\right) \stackrel{d}{\rightarrow} \mathcal{N}\left(0, v^{2}(x)\right),
$$

with $v(x)>0$. If, moreover, $\left(\beta_{n}\right)_{n \geq 1}$ is a positive sequence such that $\beta_{n} \rightarrow 0, \beta_{n} / \alpha_{n} \rightarrow 0$ and $\sqrt{n h^{p} \alpha_{n}} \varepsilon_{a}\left(1 / \beta_{n} \mid x\right) \rightarrow 0$ as $n \rightarrow \infty$, we then have

$$
\frac{\sqrt{n h_{n}^{p} \alpha_{n}}}{\log \left(\alpha_{n} / \beta_{n}\right)}\left(\frac{\widehat{\operatorname{RCTM}}_{a, n}^{W}\left(\beta_{n} \mid x\right)}{\operatorname{RCTM}_{a}\left(\beta_{n} \mid x\right)}-1\right) \stackrel{d}{\rightarrow} \mathcal{N}\left(0,(a v(x))^{2}\right)
$$


Let us also note that the asymptotic normality of

$$
\widehat{\operatorname{RVaR}}_{n}^{W}\left(\beta_{n} \mid x\right)=\widehat{\operatorname{RVaR}}_{n}\left(\alpha_{n} \mid x\right)\left(\alpha_{n} / \beta_{n}\right)^{\hat{\gamma}_{n}(x)}
$$

has been established in [9]. As a consequence, replacing $\widehat{\mathrm{RVaR}}_{n}$ by $\widehat{\mathrm{RVaR}}_{n}^{W}$ and $\widehat{\mathrm{RCTM}}_{a, n}$ by $\widehat{\mathrm{RCTM}}_{a, n}^{W}$ in (5) provides estimators for all risk measures considered in this paper adapted to arbitrary small levels. Their asymptotic normality is a simple consequence of Theorem 2 . In the next section, a procedure to select the tuning parameters $h$ and $\alpha_{n}$ is introduced and applied to the estimation of risk measures associated to extreme rainfall data.

\section{Application: Risk measures for extreme rainfall data}

The rainfall data is described in subsection 4.1. The implementation of the risk measure estimators requires the selection of two tuning parameters. An automatic procedure is proposed in subsection 4.2. Its finite sample performance is assessed on simulated data in subsection 4.3. Finally, the whole methodology is applied on the real data in subsection 4.4 .

\subsection{Problem and data description}

The behaviour and the efficiency of our estimators are illustrated on rainfall observations in the Cévennes-Vivarais region (southern part of France). This data set is provided by the French meteorological service Météo-France and consists in daily rainfalls measured at $N=523$ raingauge stations from 1958 to 2000. In this context, the variable of interest $Y$ is the daily rainfall measured in millimeters $(\mathrm{mm})$. The number of measurements at each station $t \in\{1, \ldots, N\}$ is denoted by $n_{t}$, the total number of observations being $n=\sum_{t=1}^{N} n_{t}=5,513,734$. The covariate $X$ is the three dimensional geographical location (longitude, latitude and altitude). A subset of the coordinates $S=\left\{x_{t}=\left(x_{1, t}, x_{2, t}, x_{3, t}\right) ; t=1, \ldots, N\right\}$ of the raingauge stations is depicted in Figure 3 . Extreme rainfall statistics are often used when a flood has occurred to assess the rarity of such an event. A typical problem is to estimate the amount that will fall on a day of exceptionally heavy rainfall which is expected to occur every $T$ years. Usually, hydrologists are interested in the value $T=100$ corresponding to a centenary event. Statistically speaking, the problem is to estimate the $T$-year return level which is the quantile of level $\beta=1 /(365.25 \times T)$ of the daily rainfall. The goal of this study is to go further and estimate the average rainfall over the $T$-year return level which is the RCTE of level $\beta=1 /(365.25 \times T)$.

\subsection{Tuning parameters selection}

Our estimators of risk measures depend on the two tuning parameters $h$ and $\alpha_{n}$. The choice of the bandwidth $h$, which controls the degree of smoothing, is a recurrent issue in non-parametric statistics. Similarly, in extreme-value theory, the choice of the number of upper order statistics, or equivalently $\alpha_{n}$ is of great importance since it raises a compromise between bias and variance. A high value of $\alpha_{n}$ is expected to lead to a large bias (since we move out of the distribution tail) while a small value of $\alpha_{n}$ leads to a large variance, see for instance Theorem 1 . Here, we propose a leave-one-out cross validation type procedure to select simultaneously $h$ and $\alpha_{n}$. To this end, 
let us consider $\mathcal{A}=\left\{\alpha_{1} \leq \cdots \leq \alpha_{R}\right\}$ such that $\alpha_{1}>1 / \min \left(n_{j}\right), j=1, \ldots, N, \alpha_{R}<0.1$ and $\mathcal{H}=\left\{h_{1} \leq \cdots \leq h_{M}\right\}$, such that there is at least one observation in the ball $B\left(x, h_{1}\right)$ for all $x$. The principle of the procedure is to select the empirical pair $\left(h_{e m p}, \alpha_{e m p}\right) \in \mathcal{H} \times \mathcal{A}$ for which two different estimations of the tail index $\gamma\left(x_{t}\right)$ at each station $t$ approximately coincide. The first estimator, denoted by $\hat{\gamma}_{n, t}$, is the well-known Hill estimator [19], it only depends on $\alpha_{n}$ and is uniquely based on the rainfall measures at station $t$. The second estimator denoted by $\hat{\gamma}_{n}\left(x_{t}\right)$ is the conditional tail-index estimator introduced in [9]. It depends both on $\alpha_{n}$ and $h$ and is computed on all the rainfall measures in the ball $B\left(x_{t}, h\right)$ except the measurements at the current station $t$. To summarize, the main idea is to select the pair $\left(h_{e m p}, \alpha_{e m p}\right)$ for which the local estimations $\gamma\left(x_{t}\right)$ and the predicted ones $\hat{\gamma}_{n}\left(x_{t}\right)$ using the neighbour stations are coherent. To be more specific, the algorithm is the following:

1. Loop on all pairs $\left(h_{i}, \alpha_{j}\right) \in \mathcal{H} \times \mathcal{A}$ and on all stations $t \in\{1, \ldots, N\}$.

2. Compute the Hill estimator at station $t$ with level $\alpha_{j}$ to obtain $\hat{\gamma}_{n, t, j}$.

3. Compute the conditional tail-index estimator using the measures in $B\left(x_{t}, h_{i}\right) \backslash\left\{x_{t}\right\}$ with level $\alpha_{j}$ to obtain $\hat{\gamma}_{n, i, j}\left(x_{t}\right)$.

4. Compute the distance $W_{h_{i}, \alpha_{j}}\left(x_{t}\right)=\left(\hat{\gamma}_{n, t, j}-\hat{\gamma}_{n, i, j}\left(x_{t}\right)\right)^{2}$.

5. End of the loop.

6. The optimal pair is given by

$$
\left(h_{e m p}, \alpha_{e m p}\right)=\underset{\left(h_{i}, \alpha_{j}\right) \in \mathcal{H} \times \mathcal{A}}{\arg \min } \operatorname{median}\left\{W_{h_{i}, \alpha_{j}}\left(x_{t}\right), t \in\{1, \ldots, N\}\right\} .
$$

\subsection{Validation on simulation}

The previous procedure is tested on two heavy-tailed distributions, the Fréchet distribution and the Burr distribution. The survival function of the Fréchet distribution is $\bar{F}(y \mid x)=1-\exp \left(-y^{-1 / \gamma(x)}\right)$, for $y \geq 0$, and the associated RCTE can be written

$$
\operatorname{RCTE}\left(\beta_{n} \mid x\right)=\frac{1}{\beta_{n}} \int_{0}^{\operatorname{RVaR}\left(\beta_{n} \mid x\right)^{-1 / \gamma(x)}} t^{\gamma(x)} \exp (-t) d t \text { with } \operatorname{RVaR}\left(\beta_{n} \mid x\right)=\left(-\log \left(1-\beta_{n}\right)\right)^{-\gamma(x)} .
$$

The survival function of the chosen Burr distribution is given by $\bar{F}(y \mid x)=\left(1+y^{1 / \gamma(x)}\right)^{-1}$ for $y \geq 0$, and the associated RCTE is

$$
\operatorname{RCTE}\left(\beta_{n} \mid x\right)=\mathcal{I}\left(\frac{\operatorname{RVaR}\left(\beta_{n} \mid x\right)^{-1 / \gamma(x)}}{1+\operatorname{RVaR}\left(\beta_{n} \mid x\right)^{-1 / \gamma(x)}}, 1-\gamma, 1+\gamma\right) \text { with } \operatorname{RVaR}\left(\beta_{n} \mid x\right)=\beta_{n}^{-\gamma(x)}\left(1-\beta_{n}\right)^{\gamma(x)},
$$

and where

$$
\mathcal{I}(r, p, q)=\frac{B(r, p, q)}{B(p, q)} \quad \text { with } \quad B(r, p, q)=\int_{0}^{r} w^{p-1}(1-w)^{q-1} d w
$$

being the incomplete beta function and $B(p, q)$ the beta function. In this simulation study, we choose the following conditional tail index:

$$
\gamma: x \in(0,1) \rightarrow \gamma(x)=\frac{1}{2}\left(\frac{1}{10}+\sin (\pi x)\right)\left(\frac{11}{10}-\frac{1}{2} \exp \left(-64(x-1 / 2)^{2}\right)\right) .
$$


Note that $\gamma(x)$ is close to $1 / 2$ when $x=0.3$ or $x=0.7$. Let $z_{1}, z_{2}$ and $z_{3}$ be respectively the latitude, longitude and altitude normalised in the unit interval. Two choices of covariates $x$ were used for $\gamma(x): x_{\text {euc }}:=\sqrt{\left(z_{1}^{2}+z_{2}^{2}\right) / 2}$ and $x_{\text {alt }}:=z_{3}$. The tuning parameters are selected in the sets $\mathcal{A}=\left\{1 /(6 \times 365.25), 1 /(5 \times 365.25), \ldots, 1 / 365.25,4.10^{-3}, 6.10^{-3}, \ldots, 10^{-2}, 2.10^{-2}, \ldots, 10^{-1}\right\}$ and $\mathcal{H}=\{14,15, \ldots, 30\}$. Recall that the conditional tail index estimator introduced in [9] is given by

$$
\hat{\gamma}_{n}(x)=\sum_{j=1}^{J}\left[\log \widehat{\operatorname{RVaR}}_{n}\left(\tau_{j} \alpha_{n} \mid x\right)-\log \widehat{\operatorname{RVaR}}_{n}\left(\tau_{1} \alpha_{n} \mid x\right)\right] / \sum_{j=1}^{J} \log \left(\tau_{1} / \tau_{j}\right),
$$

where $\left(\tau_{j}\right)_{j \geq 1}$ is a positive non-increasing sequence of weights. Two sequences are investigated:

1. the harmonic sequence defined for all $j=1, \ldots, J$ by $\tau_{j}^{H a}=1 / j$ with $J=9$,

2. the geometric sequence defined for all $j=1, \ldots, J$ by $\tau_{j}^{G}=(1 / j)^{(j / J)}$ with $J=15$.

In both cases, the number of terms $J$ was selected to minimize the asymptotic variance of $\hat{\gamma}_{n}(x)$. Finally, a bi-quadratic kernel was used:

$$
K(x):=K\left(z_{1}, z_{2}\right)=\frac{15}{16}\left[1-\left(z_{1}^{2}+z_{2}^{2}\right)\right]^{2} \mathbb{I}\left\{z_{1}^{2}+z_{2}^{2} \leq 1\right\} .
$$

To assess the performance of our procedure, it is compared to the Oracle (optimal) choice $\left(h_{\text {opt }}, \alpha_{\text {opt }}\right)$ which is based on the knowledge of the true tail index function:

$$
\left(h_{\text {opt }}, \alpha_{\text {opt }}\right)=\underset{\left(h_{i}, \alpha_{j}\right) \in \mathcal{H} \times \mathcal{A}}{\arg \min } \operatorname{median}\left\{V_{h_{i}, \alpha_{j}}\left(x_{t}\right), t \in\{1, \ldots, N\}\right\},
$$

where $V_{h_{i}, \alpha_{j}}\left(x_{t}\right)=\left(\gamma\left(x_{t}\right)-\hat{\gamma}_{n, i, j}\left(x_{t}\right)\right)^{2}$ is the distance to the true tail index function. The selected parameters are displayed in Table 2 . It appears that the cross-validation procedure approximately selects the same tuning parameters as the Oracle for all the considered choices of distribution, covariate and weights. Most importantly, one can observe on Figure 1 that the error distributions

\begin{tabular}{|c|c|c|c|}
\hline \multicolumn{2}{|c|}{ Burr distribution } & \multicolumn{2}{|c|}{ Fréchet distribution } \\
\hline$x_{e u c}$ and $\tau_{j}^{H a}$ & $x_{a l t}$ and $\tau_{j}^{G}$ & $x_{e u c}$ and $\tau_{j}^{G}$ & $x_{a l t}$ and $\tau_{j}^{H a}$ \\
\hline$h_{o p t}=22$ & $h_{o p t}=24$ & $h_{o p t}=22$ & $h_{o p t}=26$ \\
\hline$h_{e m p}=24$ & $h_{e m p}=22$ & $h_{e m p}=20$ & $h_{e m p}=24$ \\
\hline$\alpha_{\text {opt }}=1 / 365.25$ & $\alpha_{o p t}=1 /(365.25 \times 2)$ & $\alpha_{\text {opt }}=1 /(365.25 \times 3)$ & $\alpha_{o p t}=1 / 365.25$ \\
\hline$\alpha_{e m p}=0.001$ & $\alpha_{e m p}=1 / 365.25$ & $\alpha_{e m p}=1 / 365.25$ & $\alpha_{e m p}=0.004$ \\
\hline
\end{tabular}
on the tail index also nearly coincide. This result indicates that the cross-validation procedure is almost as efficient as the Oracle who knows the solution.

Table 2: Results of the selection procedure

Similar results can be observed on the extrapolated RCTE defined by

$$
\widehat{\operatorname{RCTE}}_{n}^{W}(\beta \mid x)=\widehat{\operatorname{RCTE}}_{n}(\alpha \mid x)\left(\frac{\alpha}{\beta}\right)^{\hat{\gamma}_{n}(x)},
$$


and computed for $\beta=1 /(365.25 \times 100)$ corresponding to a centenary rainfall. In this case, the quality of the estimation is assessed thanks to the relative error:

$$
Q_{n}(x)=\left(\frac{\widehat{\operatorname{RCTE}}_{n}^{W}(\beta \mid x)}{\operatorname{RCTE}(\beta \mid x)}-1\right)^{2} .
$$

The two histograms of $Q_{n}\left(x_{t}\right), t \in\{1, \ldots, N\}$ obtained with $\left(h_{e m p}, \alpha_{e m p}\right)$ and $\left(h_{o p t}, \alpha_{\text {opt }}\right)$ are depicted on Figure 2. Both set of parameters yield approximately the same error distribution.

\subsection{Estimated risk measures on extreme rainfalls}

The cross-validation procedure applied to the real data set with $\tau_{j}^{H a}$ yields $h_{e m p}=24$ and $\alpha_{e m p}=$ $1 /(365.25 \times 3)$. The estimated conditional tail index is then computed on a grid of $200 \times 200$ ungauged locations regularly distributed on the Cévennes-Vivarais region, see Figure 3, top panels. Using the asymptotic distribution of $\hat{\gamma}_{n}(x)$ established in [9], Corollary 2, pointwise confidence intervals can also be computed. It appears that, for a confidence level of $95 \%$, one can assume that $\gamma(x)<1 / 2$. At the opposite, the assumption that $\gamma(x)$ does not depend on $x$, i.e. $\gamma(x)$ is constant on the Cévennes-Vivarais region, cannot be accepted. It is then possible to estimate risk measures associated to a 100-year return period. Here, we focus on $\operatorname{RVaR}_{n}\left(\beta_{n} \mid x\right)$ and $\operatorname{RCTE}_{n}\left(\beta_{n} \mid x\right)$ with $\beta_{n}=1 /(365.25 \times 100)$. The associated estimators $\widehat{\mathrm{RVaR}}_{n}^{W}\left(\beta_{n} \mid x\right)$ and $\widehat{\mathrm{RCTE}}_{n}^{W}\left(\beta_{n} \mid x\right)$ are displayed on Figure 3, bottom panels. The estimated 100-year return level $\widehat{\mathrm{RVaR}}_{n}^{W}\left(\beta_{n} \mid x\right)$ is similar to the results obtained in [6] using kriging methods. More interestingly, the $\widehat{\mathrm{RCTE}}_{n}^{W}\left(\beta_{n} \mid x\right)$ can be 150 millimeters higher than the $\widehat{\mathrm{RVaR}}_{n}^{W}\left(\beta_{n} \mid x\right)$ on the mountains area.

\section{References}

[1] P. Artzner, F. Delbaen, J.M. Eber and D. Heath. Coherent measures of risk. Mathematical Finance, 9:203-228, 1999.

[2] J. Beirlant, T. de Wet and Y. Goegebeur. Nonparametric estimation of extreme conditional quantiles. Journal of statistical computation and simulation, 74:567-580, 2004.

[3] N.H. Bingham, C.M. Goldie and J.L. Teugels. Regular Variation, Cambridge University Press, 1987.

[4] V. Brazaukas, B. Jones, L. Puri and R. Zitikis. Estimating conditional tail expectation with actuarial applications in view, Journal of Statistical Planning and Inference, 128:3590-3604, 2008.

[5] J. Cai and K.S Tan. Optimal retention for a stop-loss reinsurance under the VaR and CTE risk measures, Astin Bulletin, 37(1): 93 pages, 2007.

[6] D. Ceresetti, E. Ursu, J. Carreau, S. Anquetin, J.D. Creutin, L. Gardes, S. Girard and G. Molinié. Evaluation of classical spatial-analysis schemes of extreme rainfall, Natural Hazards and Earth System Sciences, 12:3229-3240, 2012. 
[7] D. Chen, T. Mao, X. Pan and T. Hu. Extreme value behavior of aggregate dependent risks, Insurance: Mathematics and Economics, 50(1): 99-108, 2012.

[8] E. Deme, S. Girard and A. Guillou. Reduced-bias estimator of the Conditional Tail Expectation of heavy-tailed distributions, http://hal.inria.fr/hal-00823260, 2013.

[9] A. Daouia, L. Gardes, S. Girard and A. Lekina. Kernel estimators of extreme level curves, TEST, 20:311-333, 2011.

[10] A. Daouia, L. Gardes and S. Girard. On kernel smoothing for extremal quantile regression, Bernoulli, to appear, 2013.

[11] P. Embrechts, C. Klüppelberg, T. Mikosch. Modelling extremal events for insurance and finance, Springer, 1997.

[12] P. Embrechts. Extremes and integrated risk management, Risk Books, 2000.

[13] L. Gardes and S. Girard. A moving window approach for nonparametric estimation of the conditional tail index. Journal of Multivariate Analysis, 99:2368-2388, 2008.

[14] L. Gardes and S. Girard. Conditional extremes from heavy-tailed distributions: an application to the estimation of extreme rainfall return levels, Extremes, 13:177-204, 2010.

[15] L. Gardes and S. Girard. Functional kernel estimators of large conditional quantiles, Electronic Journal of Statistics, 6:1715-1744, 2012.

[16] L. Gardes, A. Guillou and A. Schorgen. Estimating the conditional tail index by integrating a kernel conditional quantile estimator, Journal of Statistical Planning and Inference, 142(6):1586-1598, 2012.

[17] L. Gardes and G. Stupfler. Estimation of the conditional tail index using a smoothed local Hill estimator, Extremes, to appear, 2013.

[18] Y. Goegebeur and T. de Wet. Local estimation of the second order parameter in extreme value statistics and local unbiased estimation of the tail index. Communications in Statistics-Theory and Methods, 41:3575-3607, 2012.

[19] B.M. Hill. A simple general approach to inference about the tail of a distribution. The Annals of Statistics, 3:1163-1174, 1975.

[20] J. Hong, and A. Elshahat. Conditional tail variance and conditional tail skewness. Journal of Financial and Economic Practice, 10(1):147-156, 2010.

[21] L. Hua and H. Joe. Second order regular variation and conditional tail expectation of multiple risks. Insurance: Mathematics and Economics, 49(3):537-546, 2011.

[22] P. Jorion. Value at risk: the new benchmark for managing financial risk, McGraw-Hill New York, 2007.

[23] Z. Landsman and E.A. Valdez. Tail conditional expectations for elliptical distributions. North American Actuarial Journal, 7:55-71, 2003. 
[24] A.J. McNeil, R. Frey, and P. Embrechts. Quantitative risk management: concepts, techniques, and tools, Princeton university press, 2005.

[25] A. Necir, A. Rassoul and R. Zitikis. Estimating the conditional tail expectation in the case of heavy-tailed losses. Journal of Probability and Statistics, ID 596839, 17 pages, 2010.

[26] A. Necir and R. Zitikis. Coupled risk measures and their empirical estimation when losses follow heavy-tailed distributions. Available at SSRN, http://ssrn. com/abstract=1855623, 2011.

[27] E. Parzen. On estimation of a probability density function and mode. The Annals of Mathematical Statistics, 33:1065-1076, 1962.

[28] S.I. Resnick. Extreme Values, Regular Variation, and Point Processes, Springer, 2008.

[29] R.T. Rockafellar, S. Uryasev. Optimization of conditional value-at-risk. Journal of Risk, 2:2142,2000 .

[30] M. Rosenblatt. Remarks on some nonparametric estimates of a density function. The Annals of Mathematical Statistics, 832-837, 1956.

[31] D. Tasche. Expected shortfall and beyond. Journal of Banking \& Finance, 26:1519-1533, 2002.

[32] E.A. Valdez. Tail conditional variance for elliptically contoured distributions. Belgian Actuarial Bulletin, 5:26-36, 2005.

[33] H. Wang and C.L. Tsai. Tail index regression. Journal of the American Statistical Association, 104:1233-1240, 2009.

[34] H. Wang, D. Li and X. He. Estimation of high conditional quantiles for heavy-tailed distributions. Journal of the American Statistical Association, 107:1453-1464, 2012.

[35] I. Weissman. Estimation of parameters and large quantiles based on the $k$ largest observations. Journal of the American Statistical Association, 73:812-815, 1978.

\section{Appendix: Proofs}

\subsection{Preliminary results}

This lemma provides an equivalent of $\varphi_{a}(y \mid x)$ when $y \rightarrow \infty$. We refer to [7, Corollary 3.2] for a similar result in the unconditional case.

Lemma 1 Under (F.1), if $y \rightarrow \infty$, then for $a \in[0,1 / \gamma(x))$,

$$
\varphi_{a}(y \mid x)=\frac{1}{1-a \gamma(x)} y^{a} \bar{F}(y \mid x)(1+o(1)) .
$$

Furthermore, under the additional condition (F.2), the derivative $\varphi_{a}^{\prime}(. \mid x)$ of the function $\varphi_{a}(. \mid x)$ exists and is a regularly varying function such that

$$
\varphi_{a}^{\prime}(y \mid x)=\frac{a \gamma(x)-1}{\gamma(x)} \frac{\varphi_{a}(y \mid x)}{y}(1+o(1)) .
$$


Proof. First, integrating by part leads to

$$
a \int_{y}^{\infty} z^{a-1} \bar{F}(z \mid x) d z=\varphi_{a}(y \mid x)-y^{a} \bar{F}(y \mid x) .
$$

Using [28, Eq. (0.32)] together with $y \rightarrow y^{a-1} \bar{F}(y \mid x) \in \mathcal{R} \mathcal{V}_{a-1 / \gamma(x)-1}, a-1 / \gamma(x)-1<-1$ and $y \rightarrow \infty$ yield

$$
\int_{y}^{\infty} z^{a-1} \bar{F}(z \mid x) d z=\frac{\gamma(x)}{1-a \gamma(x)} y^{a} \bar{F}(y \mid x)(1+o(1)) .
$$

Replacing in (9) and dividing both sides by $\frac{1}{1-a \gamma(x)} y^{a} \bar{F}(y \mid x)$ lead to

$$
\frac{\varphi_{a}(y \mid x)}{\frac{1}{1-a \gamma(x)} y^{a} \bar{F}(y \mid x)}-1+a \gamma(x)=a \gamma(x)(1+o(1)),
$$

which concludes the first part of the proof. Next, under (F.2), derivating both sides of (9) yields

$$
\varphi_{a}^{\prime}(y \mid x)=y^{a} \bar{F}^{\prime}(y \mid x)=y^{a-1} \bar{F}(y \mid x) \frac{y \bar{F}^{\prime}(y \mid x)}{\bar{F}(y \mid x)},
$$

and using [28, Corollary of Theorem 0.6], it follows that

$$
\frac{y \bar{F}^{\prime}(y \mid x)}{\bar{F}(y \mid x)}=-\frac{1}{\gamma(x)}(1+o(1))
$$

which concludes the proof.

As a consequence of Lemma 1 and (1), we obtain $\operatorname{RCTE}\left(\alpha_{n} \mid x\right) / \operatorname{RVaR}\left(\alpha_{n} \mid x\right) \sim 1 /(1-\gamma(x))$ which is an extension of the unconditional result, see for instance [21]. The second lemma is also of analytical nature. It provides a second order asymptotic expansion of the RCTM.

Lemma 2 Suppose (F.1), (F.2) and (F.3) hold and let $0<\beta_{n}<\alpha_{n}$ be two sequences such that $\alpha_{n} \rightarrow 0$ as $n \rightarrow \infty$. Then,

$$
\left|\log \operatorname{RCTM}_{a}\left(\alpha_{n} \mid x\right)-\log \operatorname{RCTM}_{a}\left(\beta_{n} \mid x\right)+a \gamma(x) \log \left(\alpha_{n} / \beta_{n}\right)\right|=O\left(\log \left(\alpha_{n} / \beta_{n}\right) \varepsilon_{a}\left(1 / \beta_{n}\right)\right) .
$$

Proof. Using (6) and (F.2), we have

$$
\log \operatorname{RCTM}_{a}\left(\alpha_{n} \mid x\right)=-a \gamma(x) \log \left(\alpha_{n}\right)+\log (c(x))+\int_{1}^{1 / \alpha_{n}} \frac{\varepsilon_{a}(u \mid x)}{u} d u,
$$

and consequently

$$
\Delta_{n}:=\log \operatorname{RCTM}_{a}\left(\alpha_{n} \mid x\right)-\log \operatorname{RCTM}_{a}\left(\beta_{n} \mid x\right)+a \gamma(x) \log \left(\alpha_{n} / \beta_{n}\right)=\int_{1 / \beta_{n}}^{1 / \alpha_{n}} \frac{\varepsilon_{a}(u \mid x)}{u} d u .
$$

From (F.3), we obtain $\left|\Delta_{n}\right| \leq\left|\varepsilon_{a}\left(1 / \beta_{n}\right)\right| \log \left(\beta_{n} / \alpha_{n}\right)$ and the conclusion follows.

Let us remark that the kernel estimator (2) of the conditional expectation can be rewritten as $\hat{\varphi}_{a, n}\left(y_{n} \mid x\right)=\hat{\psi}_{a, n}\left(y_{n} \mid x\right) / \hat{g}_{n}(x)$ where

$$
\hat{\psi}_{a, n}(y \mid x)=\frac{1}{n} \sum_{i=1}^{n} K_{h}\left(x-X_{i}\right) Y_{i}^{a} \mathbb{I}\left\{Y_{i}>y\right\},
$$

is an estimator of $\psi_{a}(y \mid x)=g(x) \varphi_{a}(y \mid x)$ and $\hat{g}_{n}(x)$ is the kernel estimator of the density $g(x)$

$$
\hat{g}_{n}(x)=\frac{1}{n} \sum_{i=1}^{n} K_{h}\left(x-X_{i}\right) .
$$


Lemma 3 Suppose (F.1), (F.2), (L) and (K) hold. Let $x \in \mathbb{R}^{p}$ such that $g(x)>0$ and $y_{n} \rightarrow \infty$ such that $n h^{p} \bar{F}\left(y_{n} \mid x\right) \rightarrow \infty$.

(i) Let $0 \leq a<1 / \gamma(x)$. If $\omega\left(x, y_{n}, a, 0, h\right) \rightarrow 0$ then

$$
\mathbb{E}\left(\hat{\psi}_{a, n}\left(y_{n} \mid x\right)\right)=\psi_{a}\left(y_{n} \mid x\right)\left(1+O(h)+O\left(\omega\left(x, y_{n}, a, 0, h\right)\right)\right) .
$$

(ii) Let $0 \leq a_{1}<\cdots<a_{J+1}<1 /(2 \gamma(x))$ where $J$ is a positive integer and consider sequences $\left(y_{n, j}\right), j=1, \ldots, J+1$ such that

$$
\max _{j \in\{1, \ldots, J+1\}}\left\{\left|\frac{y_{n, j}}{y_{n}}-1\right|\right\} \rightarrow 0 .
$$

If there exists, $\xi>0$ such that $\max _{a \in \mathcal{L}\left(\left\{a_{1}, \ldots, a_{J+1}\right\}\right)} \omega\left(x, y_{n}, a, \xi, h\right) \rightarrow 0$ then, the random vector

$$
\left\{\sqrt{n h^{p} \bar{F}\left(y_{n} \mid x\right)}\left(\frac{\hat{\psi}_{a_{j}, n}\left(y_{n, j} \mid x\right)-\mathbb{E}\left(\hat{\psi}_{a_{j}, n}\left(y_{n, j} \mid x\right)\right)}{\psi_{a_{j}}\left(y_{n, j} \mid x\right)}\right)\right\}_{j \in\{1, \ldots, J+1\}}
$$

is asymptotically Gaussian, centred, with covariance matrix $\|K\|_{2}^{2} \Sigma^{(1)}(x) / g(x)$ where

$$
\Sigma_{i, j}^{(1)}(x)=\frac{\left(1-a_{i} \gamma(x)\right)\left(1-a_{j} \gamma(x)\right)}{1-\left(a_{i}+a_{j}\right) \gamma(x)},(i, j) \in\{1, \ldots, J+1\}^{2} .
$$

Proof. (i) Since the $\left(X_{i}, Y_{i}\right), i=1, \ldots, n$ are identically distributed, it follows that

$$
\mathbb{E}\left(\hat{\psi}_{a, n}\left(y_{n} \mid x\right)\right)=\int_{\mathbb{R}^{p}} K_{h}(x-t) \varphi_{a}\left(y_{n} \mid t\right) g(t) d t=\int_{S} K(u) \varphi_{a}\left(y_{n} \mid x-h u\right) g(x-h u) d u,
$$

under $(\mathbf{K})$. Let us now consider

$$
\begin{aligned}
\left|\mathbb{E}\left(\hat{\psi}_{a, n}\left(y_{n} \mid x\right)\right)-\psi_{a}\left(y_{n} \mid x\right)\right| & \leq \varphi_{a}\left(y_{n} \mid x\right) \int_{S} K(u)|g(x-h u)-g(x)| d u \\
& +\varphi_{a}\left(y_{n} \mid x\right) \int_{S} K(u)\left|\frac{\varphi_{a}\left(y_{n} \mid x-h u\right)}{\varphi_{a}\left(y_{n} \mid x\right)}-1\right| g(x-h u) d u .
\end{aligned}
$$

Under $(\mathbf{L})$, and since $g(x)>0$, we have

$$
(11) \leq \varphi_{a}\left(y_{n} \mid x\right) c_{g} h \int_{S} d(u, 0) K(u) d u=\varphi_{a}\left(y_{n} \mid x\right) O(h) .
$$

Besides, in view of (13),

$$
\begin{aligned}
(12) & \leq \varphi_{a}\left(y_{n} \mid x\right) \omega\left(x, y_{n}, a, 0, h\right) \int_{S} K(u) g(x-h u) d u=\varphi_{a}\left(y_{n} \mid x\right) g(x) \omega\left(x, y_{n}, a, 0, h\right)(1+o(1)) \\
& \leq \psi_{a}\left(y_{n} \mid x\right) \omega\left(x, y_{n}, a, 0, h\right)(1+o(1)) .
\end{aligned}
$$

Combining (13) and (14) concludes the first part of the proof. 
(ii) Let $\beta \neq 0$ in $\mathbb{R}^{J+1}, \Lambda_{n}(x)=\left(n h^{p} \psi_{0}\left(y_{n} \mid x\right)\right)^{-1 / 2}$, and consider the random variable

$$
\begin{aligned}
\Psi_{n} & =\sum_{j=1}^{J+1} \beta_{j}\left(\frac{\hat{\psi}_{a_{j}, n}\left(y_{n, j} \mid x\right)-\mathbb{E}\left(\hat{\psi}_{a_{j}, n}\left(y_{n, j} \mid x\right)\right)}{\Lambda_{n}(x) \psi_{a_{j}}\left(y_{n, j} \mid x\right)}\right), \\
& =\sum_{i=1}^{n} \frac{1}{n \Lambda_{n}(x)}\left\{\sum_{j=1}^{J+1} \frac{\beta_{j} K_{h}\left(x-X_{i}\right) Y_{i}^{a_{j}} \mathbb{I}\left\{Y_{i} \geq y_{n, j}\right\}}{\psi_{a_{j}}\left(y_{n, j} \mid x\right)}\right. \\
& \left.-\mathbb{E}\left(\sum_{j=1}^{J+1} \frac{\beta_{j} K_{h}(x-X) Y^{a_{j}} \mathbb{I}\left\{Y \geq y_{n, j}\right\}}{\psi_{a_{j}}\left(y_{n, j} \mid x\right)}\right)\right\}, \\
& =: \sum_{i=1}^{n} Z_{i, n} .
\end{aligned}
$$

Clearly, $\left\{Z_{i, n}, i=1, \ldots, n\right\}$ is a set of centred, independent and identically distributed random variables with variance

$$
\operatorname{var}\left(Z_{1, n}\right)=\frac{1}{n^{2} h^{2 p} \Lambda_{n}^{2}(x)} \operatorname{var}\left(\sum_{j=1}^{J+1} \beta_{j} K\left(\frac{x-X}{h}\right) \frac{Y^{a_{j}} \mathbb{I}\left\{Y \geq y_{n, j}\right\}}{\psi_{a_{j}}\left(y_{n, j} \mid x\right)}\right)=\frac{1}{n^{2} h^{p} \Lambda_{n}^{2}(x)} \beta^{t} B \beta,
$$

where $B$ is the $(J+1) \times(J+1)$ covariance matrix defined by

$$
B_{j, l}=\frac{A_{j, l}}{\psi_{a_{j}}\left(y_{n, j} \mid x\right) \psi_{a_{l}}\left(y_{n, l} \mid x\right)},
$$

for all $(j, l) \in\{1, \ldots, J+1\}^{2}$ and

$$
\begin{aligned}
A_{j, l} & =\frac{1}{h^{p}} \operatorname{cov}\left(K\left(\frac{x-X}{h}\right) Y^{a_{j}} \mathbb{I}\left\{Y \geq y_{n, j}\right\}, K\left(\frac{x-X}{h}\right) Y^{a_{l}} \mathbb{I}\left\{Y \geq y_{n, l}\right\}\right), \\
& =\|K\|_{2}^{2} \mathbb{E}\left(\frac{1}{h^{p}} Q\left(\frac{x-X}{h}\right) Y^{a_{j}+a_{l}} \mathbb{I}\left\{Y \geq y_{n, j} \vee y_{n, l}\right\}\right) \\
& -h^{p} \mathbb{E}\left(K_{h}(x-X) Y^{a_{j}} \mathbb{I}\left\{Y \geq y_{n, j}\right\}\right) \mathbb{E}\left(K_{h}(x-X) Y^{a_{l}} \mathbb{I}\left\{Y \geq y_{n, l}\right\}\right),
\end{aligned}
$$

with $Q():.=K^{2}(.) /\|K\|_{2}^{2}$ also satisfying assumption (K). One can use part (i) of the proof to obtain

$$
\begin{aligned}
A_{j, l} & =\|K\|_{2}^{2} \psi_{a_{j}+a_{l}}\left(y_{n, j} \vee y_{n, l} \mid x\right)\left(1+O(h)+O\left(\omega\left(x, y_{n, j} \vee y_{n, l}, a_{j}+a_{l}, 0, h\right)\right)\right) \\
& -h^{p} \psi_{a_{j}}\left(y_{n, j} \mid x\right) \psi_{a_{l}}\left(y_{n, l} \mid x\right)\left(1+O(h)+O\left(\omega\left(x, y_{n, j}, a_{j}, 0, h\right)\right)\right)\left(1+O(h)+O\left(\omega\left(x, y_{n, l}, a_{l}, 0, h\right)\right)\right) .
\end{aligned}
$$

Let $\xi>0$ such that $\max _{a \in \mathcal{L}\left(\left\{a_{1}, \ldots, a_{J+1}\right\}\right)} \omega\left(x, y_{n}, a, \xi, h\right) \rightarrow 0$. Remarking that $\omega\left(x, y_{n, j}, a_{j}, 0, h\right) \leq$ $\omega\left(x, y_{n}, a_{j}, \xi, h\right)$ for $n$ large enough, we obtain

$$
\begin{aligned}
A_{j, l} & =\|K\|_{2}^{2} \psi_{a_{j}+a_{l}}\left(y_{n, j} \vee y_{n, l} \mid x\right)\left(1+O(h)+O\left(\omega\left(x, y_{n}, a_{j}+a_{l}, \xi, h\right)\right)\right) \\
& -h^{p} \psi_{a_{j}}\left(y_{n, j} \mid x\right) \psi_{a_{l}}\left(y_{n, l} \mid x\right)\left(1+O(h)+O\left(\omega\left(x, y_{n}, a_{j}, \xi, h\right)\right)\right)\left(1+O(h)+O\left(\omega\left(x, y_{n}, a_{l}, \xi, h\right)\right)\right) .
\end{aligned}
$$

Now,

$$
\max \left(\omega\left(x, y_{n}, a_{j}, \xi, h\right), \omega\left(x, y_{n}, a_{l}, \xi, h\right), \omega\left(x, y_{n}, a_{j}+a_{l}, \xi, h\right)\right) \leq \max _{a \in \mathcal{L}\left(\left\{a_{1}, \ldots, a_{J+1}\right\}\right)} \omega\left(x, y_{n}, a, \xi, h\right),
$$


leads to

$$
\begin{aligned}
B_{j, l} & =\frac{\|K\|_{2}^{2} \psi_{a_{j}+a_{l}}\left(y_{n, j} \vee y_{n, l} \mid x\right)}{\psi_{a_{j}}\left(y_{n, j} \mid x\right) \psi_{a_{l}}\left(y_{n, l} \mid x\right)}\left[1+O(h)+O\left(\max _{a \in \mathcal{L}\left(\left\{a_{1}, \ldots, a_{J+1}\right\}\right)} \omega\left(x, y_{n}, a, \xi, h\right)\right)\right] \\
& -h^{p}\left[1+O(h)+O\left(\max _{a \in \mathcal{L}\left(\left\{a_{1}, \ldots, a_{J+1}\right\}\right)} \omega\left(x, y_{n}, a, \xi, h\right)\right)\right] .
\end{aligned}
$$

Let us recall that, since $\psi_{a}(. \mid x)$ is regularly varying, it follows that $\psi_{a_{j}}\left(y_{n, j} \mid x\right) \sim \psi_{a_{j}}\left(y_{n} \mid x\right) \rightarrow 0$ for all $j \in\{1, \ldots, J+1\}$. Lemma 1 thus entails

$$
B_{j, l}=\frac{\|K\|_{2}^{2}}{\psi_{0}\left(y_{n} \mid x\right)} \frac{\left(1-a_{j} \gamma(x)\right)\left(1-a_{l} \gamma(x)\right)}{1-\left(a_{j}+a_{l}\right) \gamma(x)}(1+o(1))=\frac{\|K\|_{2}^{2}}{\psi_{0}\left(y_{n} \mid x\right)} \Sigma_{j, l}^{(1)}(x)(1+o(1)) .
$$

Therefore, $\operatorname{var}\left(Z_{1, n}\right) \sim\|K\|_{2}^{2} \beta^{t} \Sigma^{(1)}(x) \beta / n$. As a preliminary conclusion, the variance of $\Psi_{n}$ converges to $\|K\|_{2}^{2} \beta^{t} \Sigma^{(1)}(x) \beta$. Consequently, using Lyapounov theorem for the asymptotic normality of sums of triangular arrays, it remains to prove that there exists $\eta>0$ such that:

$$
\sum_{i=1}^{n} \mathbb{E}\left|Z_{i, n}\right|^{2+\eta}=n \mathbb{E}\left|Z_{1, n}\right|^{2+\eta} \rightarrow 0 .
$$

Straightforward calculations lead to

$$
\begin{aligned}
\mathbb{E}\left|Z_{1, n}\right|^{2+\eta} & =\left(\frac{1}{n \Lambda_{n}(x)}\right)^{2+\eta} \mathbb{E} \mid \sum_{j=1}^{J+1} \frac{\beta_{j} K_{h}(x-X) Y^{a_{j}} \mathbb{I}\left\{Y \geq y_{n, j}\right\}}{\psi_{a_{j}}\left(y_{n, j} \mid x\right)} \\
& -\left.\mathbb{E}\left(\sum_{j=0}^{J+1} \frac{\beta_{j} K_{h}(x-X) Y^{a_{j}} \mathbb{I}\left\{Y \geq y_{n, j}\right\}}{\psi_{a_{j}}\left(y_{n, j} \mid x\right)}\right)\right|^{2+\eta} .
\end{aligned}
$$

Besides, for every pair of random variables $\left(T_{1}, T_{2}\right)$ with finite $(2+\eta)$ th order moments, one has

$$
\mathbb{E}\left(\left|T_{1}+T_{2}\right|^{2+\eta}\right) \leq 2^{2+\eta} \max _{i=\{1,2\}} \mathbb{E}\left(\left|T_{i}\right|^{2+\eta}\right),
$$

leading to

$$
\mathbb{E}\left|Z_{1, n}\right|^{2+\eta} \leq\left(\frac{2}{n \Lambda_{n}(x)}\right)^{2+\eta} \mathbb{E}\left|\sum_{j=1}^{J+1} \frac{\beta_{j} K_{h}(x-X) Y^{a_{j}} \mathbb{I}\left\{Y \geq y_{n, j}\right\}}{\psi_{a_{j}}\left(y_{n, j} \mid x\right)}\right|^{2+\eta} .
$$

Lemma 1 and $y_{n, j}=y_{n}(1+o(1))$ for all $j \in\{1, \ldots, J+1\}$ yield

$$
\begin{aligned}
\mathbb{E}\left|Z_{1, n}\right|^{2+\eta} & \leq\left(\frac{2}{n \Lambda_{n}(x) \psi_{0}\left(y_{n} \mid x\right)}\right)^{2+\eta} \\
& \times \mathbb{E}\left|\sum_{j=1}^{J+1} \beta_{j} K_{h}(x-X) \mathbb{I}\left\{Y \geq y_{n, j}\right\}\left(\frac{Y}{y_{n, j}}\right)^{a_{j}}\left(1-a_{j} \gamma(x)\right)\right|^{2+\eta}(1+o(1)) .
\end{aligned}
$$

Letting $\tilde{a}=\max \left\{a_{1}, \ldots, a_{J+1}\right\}$ and $\tilde{y}_{n}=\min \left\{y_{n, 1}, \ldots, y_{n, J+1}\right\}$, it follows that for $n$ large enough,

$$
n \mathbb{E}\left|Z_{1, n}\right|^{2+\eta} \leq 2 n\left(\frac{2(1-\tilde{a} \gamma(x))}{n h^{p} \Lambda_{n}(x) \psi_{0}\left(y_{n} \mid x\right) \tilde{y} \tilde{a}}\right)^{2+\eta} \sum_{j=1}^{J+1}\left|\beta_{j}\right|^{2+\eta} \mathbb{E}\left(K\left(\frac{x-X}{h}\right) Y^{\tilde{a}} \mathbb{I}\left\{Y \geq \tilde{y}_{n}\right\}\right)^{2+\eta} .
$$


Choosing $\eta$ such that $0<\eta<-2+1 /(\tilde{a} \gamma(x))$, (i) implies that

$$
\begin{aligned}
\mathbb{E}\left(K\left(\frac{x-X}{h}\right) Y^{\tilde{a}} \mathbb{I}\left\{Y \geq \tilde{y}_{n}\right\}\right)^{2+\eta} & =h^{p}\|K\|_{2+\eta}^{2+\eta} \mathbb{E}\left(N_{h}(x-X) Y^{\tilde{a}(2+\eta)} \mathbb{I}\left\{Y \geq \tilde{y}_{n}\right\}\right), \\
& =h^{p}\|K\|_{2+\eta}^{2+\eta} \psi_{\tilde{a}(2+\eta)}\left(\tilde{y}_{n} \mid x\right)(1+o(1)),
\end{aligned}
$$

since $N():.=K^{2+\eta}(.) /\|K\|_{2+\eta}^{2+\eta}$ also fulfils assumption (K). Using Lemma 1 and the fact that $\tilde{y}_{n}=y_{n}(1+o(1))$, we obtain $n \mathbb{E}\left|Z_{1, n}\right|^{2+\eta}=O\left(\Lambda_{n}^{\eta}(x)\right) \rightarrow 0$ as $n \rightarrow \infty$ which concludes the proof.

The asymptotic behaviors of the estimators $\hat{\varphi}_{a, n}(. \mid x)$ and $\hat{\varphi}_{a, n}^{\leftarrow}(. \mid x)$ are established in the following two propositions.

Proposition 1 Suppose (F.1), (F.2), (L) and (K) hold. Let $x \in \mathbb{R}^{p}$ such that $g(x)>0$ and $0 \leq a_{1}<\cdots<a_{J+1}<1 /(2 \gamma(x))$ where $J$ is a positive integer. Consider $y_{n} \rightarrow \infty$ such that $n h^{p} \bar{F}\left(y_{n} \mid x\right) \rightarrow \infty$ as $n \rightarrow \infty$ and sequences $\left(y_{n, j}\right), j \in\{1, \ldots, J+1\}$ such that

$$
\max _{j \in\{1, \ldots, J+1\}}\left|\frac{y_{n, j}}{y_{n}}-1\right| \rightarrow 0 .
$$

If there exists $\xi>0$ such that $n h^{p} \bar{F}\left(y_{n} \mid x\right)\left(h \vee \max _{a \in \mathcal{L}\left(\left\{a_{1}, \ldots, a_{J+1}\right\}\right)} \omega\left(x, y_{n}, a, \xi, h\right)\right)^{2} \rightarrow 0$ then, the random vector

$$
\left\{\sqrt{n h^{p} \bar{F}\left(y_{n} \mid x\right)}\left(\frac{\hat{\varphi}_{a_{j}, n}\left(y_{n, j} \mid x\right)}{\varphi_{a_{j}}\left(y_{n, j} \mid x\right)}-1\right)\right\}_{j \in\{1, \ldots, J+1\}}
$$

is asymptotically Gaussian, centred, with covariance matrix $\|K\|_{2}^{2} \Sigma^{(1)}(x) / g(x)$.

Proof. Keeping in mind the notations of Lemma 3, the following expansion holds

$$
\Lambda_{n}^{-1}(x) \sum_{j=1}^{J+1} \beta_{j}\left(\frac{\hat{\varphi}_{a_{j}, n}\left(y_{n, j} \mid x\right)}{\varphi_{a_{j}}\left(y_{n, j} \mid x\right)}-1\right)=\frac{\Delta_{1, n}+\Delta_{2, n}-\Delta_{3, n}}{\hat{g}_{n}(x)},
$$

where

$$
\begin{aligned}
& \Delta_{1, n}=g(x) \Lambda_{n}^{-1}(x) \sum_{j=1}^{J+1} \beta_{j}\left(\frac{\hat{\psi}_{a_{j}, n}\left(y_{n, j} \mid x\right)-\mathbb{E}\left(\hat{\psi}_{a_{j}, n}\left(y_{n, j} \mid x\right)\right)}{\psi_{a_{j}}\left(y_{n, j} \mid x\right)}\right), \\
& \Delta_{2, n}=g(x) \Lambda_{n}^{-1}(x) \sum_{j=1}^{J+1} \beta_{j}\left(\frac{\mathbb{E}\left(\hat{\psi}_{a_{j}, n}\left(y_{n, j} \mid x\right)\right)-\psi_{a_{j}}\left(y_{n, j} \mid x\right)}{\psi_{a_{j}}\left(y_{n, j} \mid x\right)}\right), \\
& \Delta_{3, n}=\left(\sum_{j=1}^{J+1} \beta_{j}\right) \Lambda_{n}^{-1}(x)\left(\hat{g}_{n}(x)-g(x)\right) .
\end{aligned}
$$

Thus, from Lemma $3(\mathrm{ii})$, the random term $\Delta_{1, n}$ can be rewritten as

$$
\Delta_{1, n}=g(x)\|K\|_{2} \sqrt{\beta^{t} \Sigma^{(1)}(x) \beta} \xi_{n},
$$

where $\xi_{n}$ converges to a standard Gaussian random variable. The non-random term $\Delta_{2, n}$ is controlled with Lemma 3(i):

$$
\begin{aligned}
\Delta_{2, n} & =O\left(h \Lambda_{n}^{-1}(x)\right)+O\left(\Lambda_{n}^{-1}(x) \max _{a \in \mathcal{L}\left(\left\{a_{1}, \ldots, a_{J+1}\right\}\right)} \omega\left(x, y_{n}, a, \xi, h\right)\right) \\
& =O\left(n h^{p+2} \bar{F}\left(y_{n} \mid x\right)\right)^{1 / 2}+O\left(n h^{p} \bar{F}\left(y_{n} \mid x\right) \max _{a \in \mathcal{L}\left(\left\{a_{1}, \ldots, a_{J+1}\right\}\right)} \omega^{2}\left(x, y_{n}, a, \xi, h\right)\right)^{1 / 2} \\
& =o(1) .
\end{aligned}
$$


Finally, $\Delta_{3, n}$ is a classical term in kernel density estimation, which can be bounded by [9], Lemma 4 :

$$
\begin{aligned}
\Delta_{3, n} & =O\left(h \Lambda_{n}^{-1}(x)\right)+O_{P}\left(\Lambda_{n}^{-1}(x)\left(n h^{p}\right)^{-1 / 2}\right), \\
& =O\left(n h^{p+2} \bar{F}\left(y_{n} \mid x\right)\right)^{1 / 2}+O_{P}\left(\bar{F}\left(y_{n} \mid x\right)\right)^{1 / 2}=o_{P}(1) .
\end{aligned}
$$

Collecting (15)-(18), it follows that

$$
\hat{g}_{n}(x) \Lambda_{n}^{-1}(x) \sum_{j=1}^{J+1} \beta_{j}\left(\frac{\hat{\varphi}_{a_{j}, n}\left(y_{n, j} \mid x\right)}{\varphi_{a_{j}}\left(y_{n, j} \mid x\right)}-1\right)=g(x)\|K\|_{2} \sqrt{\beta^{t} \Sigma^{(1)}(x) \beta} \xi_{n}+o_{P}(1) .
$$

Finally, $\hat{g}_{n}(x) \stackrel{P}{\longrightarrow} g(x)$ yields

$$
\sqrt{n h^{p} \bar{F}\left(y_{n} \mid x\right)} \sum_{j=1}^{J+1} \beta_{j}\left(\frac{\hat{\varphi}_{a_{j}, n}\left(y_{n, j} \mid x\right)}{\varphi_{a_{j}}\left(y_{n, j} \mid x\right)}-1\right)=\|K\|_{2} \sqrt{\frac{\beta^{t} \Sigma^{(1)}(x) \beta}{g(x)}} \xi_{n}+o_{P}(1),
$$

and the result is proved.

Proposition 2 Suppose (F.1), (F.2), (L) and (K) hold. Let $x \in \mathbb{R}^{p}$ such that $g(x)>0$ and let $0 \leq a_{1}<\cdots<a_{J+1}<1 /(2 \gamma(x))$ where $J$ is a positive integer. Consider $\alpha_{n} \rightarrow 0$ such that $n h^{p} \alpha_{n} \rightarrow \infty$ as $n \rightarrow \infty$. Let $\left(\alpha_{n, j}\right), j=1, \ldots, J+1$ be sequences such that

$$
\max _{j \in\{1, \ldots, J+1\}}\left|\frac{\varphi_{a_{j}}^{\leftarrow}\left(\alpha_{n, j} \mid x\right)}{\varphi_{0}^{\leftarrow}\left(\alpha_{n} \mid x\right)}-1\right| \rightarrow 0
$$

If there exists $\xi>0$ such that $n h^{p} \alpha_{n}\left(h \vee \max _{a \in \mathcal{L}\left(\left\{a_{1}, \ldots, a_{J+1}\right\}\right)} \omega\left(x, \varphi_{0}^{\leftarrow}\left(\alpha_{n} \mid x\right), a, \xi, h\right)\right)^{2} \rightarrow 0$ then, the random vector

$$
\left\{\sqrt{n h^{p} \alpha_{n}}\left(\frac{\hat{\varphi}_{a_{j}, n}^{\leftarrow}\left(\alpha_{n, j} \mid x\right)}{\varphi_{a_{j}}^{\leftarrow}\left(\alpha_{n, j} \mid x\right)}-1\right)\right\}_{j \in\{1, \ldots, J+1\}}
$$

is asymptotically Gaussian, centred, with covariance matrix $\|K\|_{2}^{2} \Sigma^{(2)}(x) / g(x)$ where

$$
\Sigma_{i, j}^{(2)}(x)=\frac{\gamma^{2}(x)}{1-\left(a_{i}+a_{j}\right) \gamma(x)},(i, j) \in\{1, \ldots, J+1\}^{2} .
$$

Proof. Introduce for $j \in\{1, \ldots, J+1\}$,

$$
\begin{aligned}
\sigma_{n, j}(x) & =\varphi_{a_{j}}^{\leftarrow}\left(\alpha_{n, j} \mid x\right)\left(n h^{p} \alpha_{n}\right)^{-1 / 2}, \\
v_{n, j}(x) & =\alpha_{n, j}^{-1} \frac{\gamma(x)}{1-a_{j} \gamma(x)}\left(n h^{p} \alpha_{n}\right)^{1 / 2}, \\
W_{n, j}(x) & \left.=v_{n, j}(x)\left(\hat{\varphi}_{a_{j}}\left(\varphi_{a_{j}}^{\leftarrow}\left(\alpha_{n, j} \mid x\right)+\sigma_{n, j}(x) z_{j}\right) \mid x\right)-\varphi_{a_{j}}\left(\varphi_{a_{j}}^{\leftarrow}\left(\alpha_{n, j} \mid x\right)+\sigma_{n, j}(x) z_{j}\right) \mid x\right), \\
t_{n, j}(x) & =v_{n, j}(x)\left(\alpha_{n, j}-\varphi_{a_{j}}\left(\varphi_{a_{j}}^{\leftarrow}\left(\alpha_{n, j} \mid x\right)+\sigma_{n, j}(x) z_{j}\right) \mid x\right),
\end{aligned}
$$

where $\left(z_{1}, \ldots, z_{J+1}\right) \in \mathbb{R}^{J+1}$. We examine the asymptotic behavior of the cumulative distribution function defined by

$$
\begin{aligned}
\Phi_{n}\left(z_{1}, \ldots, z_{J+1}\right) & =\mathbb{P}\left(\bigcap_{j=1}^{J+1}\left\{\sigma_{n, j}^{-1}(x)\left(\hat{\varphi}_{a_{j}, n}^{\leftarrow}\left(\alpha_{n, j} \mid x\right)-\varphi_{a_{j}}^{\leftarrow}\left(\alpha_{n, j} \mid x\right)\right) \leq z_{j}\right\}\right) \\
& =\mathbb{P}\left(\bigcap_{j=1}^{J+1}\left\{W_{n, j}(x) \leq t_{n, j}(x)\right\}\right) .
\end{aligned}
$$


Let us first focus on the non-random terms $t_{n, j}(x), j \in\{1, \ldots, J+1\}$. From Lemma 1, for all $a \in[0,1 /(2 \gamma(x)))$, the function $\varphi_{a}(. \mid x)$ is differentiable and thus, for each $j \in\{1, \ldots, J+1\}$ there exists $\theta_{n, j} \in(0,1)$ such that

$$
\varphi_{a_{j}}\left(\varphi_{a_{j}}^{\leftarrow}\left(\alpha_{n, j} \mid x\right) \mid x\right)-\varphi_{a_{j}}\left(\varphi_{a_{j}}^{\leftarrow}\left(\alpha_{n, j} \mid x\right)+\sigma_{n, j}(x) z_{j} \mid x\right)=-\sigma_{n, j}(x) z_{j} \varphi_{a_{j}}^{\prime}\left(r_{n, j} \mid x\right)
$$

where $r_{n, j}=\varphi_{a_{j}}^{\leftarrow}\left(\alpha_{n, j} \mid x\right)+\theta_{n, j} \sigma_{n, j}(x) z_{j}$. It is thus clear that $r_{n, j} \sim \varphi_{a_{j}}^{\leftarrow}\left(\alpha_{n, j} \mid x\right) \rightarrow \infty$ and Lemma 1 yields

$$
\varphi_{a_{j}}^{\prime}\left(r_{n, j} \mid x\right)=\frac{\left(a_{j} \gamma(x)-1\right) \alpha_{n, j}}{\gamma(x) \varphi_{a_{j}}^{\leftarrow}\left(\alpha_{n, j} \mid x\right)}(1+o(1))
$$

In view of (19) and (20), we end up with

$$
t_{n, j}(x)=\frac{\left(1-a_{j} \gamma(x)\right) v_{n, j}(x) \sigma_{n, j}(x) \alpha_{n, j} z_{j}}{\gamma(x) \varphi_{a_{j}}^{\leftarrow}\left(\alpha_{n, j} \mid x\right)}(1+o(1))=z_{j}(1+o(1))
$$

Let us now turn to the random terms $W_{n, j}(x), j \in\{1, \ldots, J+1\}$. Clearly, sequences $y_{n, j}:=$ $\varphi_{a_{j}}^{\leftarrow}\left(\alpha_{n, j} \mid x\right)+\sigma_{n, j}(x) z_{j}, j=1, \ldots, J+1$ and $y_{n}:=\varphi_{0}^{\leftarrow}\left(\alpha_{n} \mid x\right)$ satisfy the assumptions of Proposition 1 and consequently,

$$
W_{n, j}(x)=\frac{\gamma(x)}{1-a_{j} \gamma(x)} \frac{\varphi_{a_{j}}\left(\varphi_{a_{j}}^{\leftarrow}\left(\alpha_{n, j} \mid x\right)+\sigma_{n, j}(x) z_{j} \mid x\right)}{\alpha_{n, j}}\left(n h^{p} \alpha_{n}\right)^{1 / 2}\left(\frac{\hat{\varphi}_{a_{j}}\left(y_{n, j} \mid x\right)}{\varphi_{a_{j}}\left(y_{n, j} \mid x\right)}-1\right) .
$$

Moreover, since $\varphi_{a}(. \mid x)$ is regularly varying, the following equivalences hold,

$$
\frac{\varphi_{a_{j}}\left(\varphi_{a_{j}}^{\leftarrow}\left(\alpha_{n, j} \mid x\right)+\sigma_{n, j}(x) z_{j} \mid x\right)}{\alpha_{n, j}}=\frac{\varphi_{a_{j}}\left(\varphi_{a_{j}}^{\leftarrow}\left(\alpha_{n, j} \mid x\right)\left(1+o_{\mathbb{P}}(1)\right) \mid x\right)}{\alpha_{n, j}}=1+o_{\mathbb{P}}(1) .
$$

As a consequence of Slutsky's theorem, the random vector $\left(W_{n, 1}, \ldots, W_{n, J+1}\right)$ is equal to $A(x) \xi_{n}$ where

$$
A(x)=\operatorname{diag}\left(\frac{\gamma(x)}{1-a_{1} \gamma(x)}, \ldots, \frac{\gamma(x)}{1-a_{J+1} \gamma(x)}\right),
$$

and $\xi_{n}$ is a $(J+1)$-random vector converging to a centred Gaussian random variable with covariance matrix $\|K\|_{2}^{2} \Sigma^{(1)}(x) / g(x)$. Taking account of $(21)$, we obtain that $\Phi_{n}\left(z_{1}, \ldots, z_{J+1}\right)$ converges to the cumulative distribution function of a centred Gaussian distribution with covariance matrix $\|K\|_{2}^{2} A(x) \Sigma^{(1)}(x) A(x) / g(x)=\|K\|_{2}^{2} \Sigma^{(2)}(x) / g(x)$ which is the desired result.

\subsection{Proofs of main results}

Proof of Theorem 1. Let us introduce for $j \in\{1, \ldots, J\}$,

$$
\begin{aligned}
v_{n, j}(x) & =\frac{\left(1-a_{j} \gamma(x)\right)\left(n h^{p} \alpha_{n}\right)^{1 / 2}}{\gamma(x) \varphi_{0}^{\leftarrow}\left(\alpha_{n} \mid x\right)}, \sigma_{n, j}(x)=\varphi_{a_{j}}\left(\varphi_{0}^{\leftarrow}\left(\alpha_{n} \mid x\right) \mid x\right)\left(n h^{p} \alpha_{n}\right)^{-1 / 2}, \\
\sigma_{n, 0}(x) & =\varphi_{0}^{\leftarrow}\left(\alpha_{n} \mid x\right)\left(n h^{p} \alpha_{n}\right)^{-1 / 2}, \\
t_{n, j} & =v_{n, j}(x)\left(\varphi_{0}^{\leftarrow}\left(\alpha_{n} \mid x\right)-\varphi_{a_{j}}^{\leftarrow}\left(\varphi_{a_{j}}\left(\varphi_{0}^{\leftarrow}\left(\alpha_{n} \mid x\right) \mid x\right)+\sigma_{n, j}(x) z_{j} \mid x\right)\right), \\
W_{n, j}(x) & =v_{n, j}(x)\left(\hat{\varphi}_{a_{j}, n}^{\leftarrow}\left(\varphi_{a_{j}}\left(\varphi_{0}^{\leftarrow}\left(\alpha_{n} \mid x\right) \mid x\right)+\sigma_{n, j}(x) z_{j} \mid x\right)\right. \\
& \left.-\varphi_{a_{j}}^{\leftarrow}\left(\varphi_{a_{j}}\left(\varphi_{0}^{\leftarrow}\left(\alpha_{n} \mid x\right) \mid x\right)+\sigma_{n, j}(x) z_{j} \mid x\right)\right), \\
W_{n, j}^{(0)}(x) & =v_{n, j}(x)\left(\hat{\varphi}_{0, n}^{\leftarrow}\left(\alpha_{n} \mid x\right)-\varphi_{0}^{\leftarrow}\left(\alpha_{n} \mid x\right)\right), W_{n, 0}^{(0)}(x)=\sigma_{n, 0}^{-1}(x)\left(\hat{\varphi}_{0, n}^{\leftarrow}\left(\alpha_{n} \mid x\right)-\varphi_{0}^{\leftarrow}\left(\alpha_{n} \mid x\right)\right),
\end{aligned}
$$


where $\left(z_{0}, z_{1}, \ldots, z_{J}\right) \in \mathbb{R}^{J+1}$. We examine the asymptotic behavior of the cumulative distribution function defined by

$$
\begin{aligned}
& \Phi_{n}\left(z_{0}, z_{1}, \ldots, z_{J}\right) \\
= & \mathbb{P}\left(\left\{\bigcap_{j=1}^{J}\left\{\sigma_{n, j}^{-1}(x)\left(\hat{\varphi}_{a_{j}, n}\left(\hat{\varphi}_{0, n}^{\leftarrow}\left(\alpha_{n} \mid x\right) \mid x\right)-\varphi_{a_{j}}\left(\varphi_{0}^{\leftarrow}\left(\alpha_{n} \mid x\right) \mid x\right)\right) \leq z_{j}\right\}\right\} \bigcap\left\{W_{n, 0}^{(0)}(x) \leq z_{0}\right\}\right), \\
= & \mathbb{P}\left(\left\{\bigcap_{j=1}^{J}\left\{W_{n, j}(x)-W_{n, j}^{(0)}(x) \leq t_{n, j}\right\}\right\} \bigcap\left\{W_{n, 0}^{(0)}(x) \leq z_{0}\right\}\right) .
\end{aligned}
$$

Let us first focus on the non-random terms $t_{n, j}(x), j=1, \ldots, J$. From Lemma 1 , for all $a \in$ $[0,1 /(2 \gamma(x))), \varphi_{a}^{\leftarrow}(. \mid x)$ is a differentiable regularly varying function such that

$$
\left(\varphi_{a}^{\leftarrow}\right)^{\prime}\left(y_{n} \mid x\right)=\frac{1}{\varphi_{a}^{\prime}\left(\varphi_{a}^{\leftarrow}\left(y_{n} \mid x\right) \mid x\right)}=\frac{\gamma(x) \varphi_{a}^{\leftarrow}\left(y_{n} \mid x\right)}{(a \gamma(x)-1) y_{n}}(1+o(1))
$$

as $n \rightarrow \infty$. For all $j \in\{1, \ldots, J\}$, a first order Taylor expansion leads to:

$$
\varphi_{a_{j}}^{\leftarrow}\left(\varphi_{a_{j}}\left(\varphi_{0}^{\leftarrow}\left(\alpha_{n} \mid x\right) \mid x\right) \mid x\right)-\varphi_{a_{j}}^{\leftarrow}\left(\varphi_{a_{j}}\left(\varphi_{0}^{\overleftarrow{ }}\left(\alpha_{n} \mid x\right) \mid x\right)+\sigma_{n, j}(x) z_{j} \mid x\right)=-\sigma_{n, j}(x) z_{j} q_{n, j}(x)
$$

where $q_{n, j}(x)=\left(\varphi_{a_{j}}^{\leftarrow}\right)^{\prime}\left(\varphi_{a_{j}}\left(\varphi_{0}^{\leftarrow}\left(\alpha_{n} \mid x\right) \mid x\right)+\theta_{n, j} \sigma_{n, j}(x) z_{j} \mid x\right)$ with $\left(\theta_{n, 1}, \ldots, \theta_{n, J}\right) \in(0,1)^{J}$. Since $\sigma_{n, j}(x) / \varphi_{a_{j}}\left(\varphi_{0}^{\leftarrow}\left(\alpha_{n} \mid x\right) \mid x\right)=\left(n h^{p} \alpha_{n}\right)^{-1 / 2} \rightarrow 0$ as $n \rightarrow \infty,(22)$ entails that

$$
q_{n, j}(x)=\frac{\gamma(x) \varphi_{0}^{\leftarrow}\left(\alpha_{n} \mid x\right)}{\left(a_{j} \gamma(x)-1\right) \varphi_{a_{j}}\left(\varphi_{0}^{\leftarrow}\left(\alpha_{n} \mid x\right) \mid x\right)}(1+o(1))
$$

Hence,

$$
\varphi_{a_{j}}^{\leftarrow}\left(\varphi_{a_{j}}\left(\varphi_{0}^{\overleftarrow{ }}\left(\alpha_{n} \mid x\right) \mid x\right) \mid x\right)-\varphi_{a_{j}}^{\leftarrow}\left(\varphi_{a_{j}}\left(\varphi_{0}^{\overleftarrow{ }}\left(\alpha_{n} \mid x\right) \mid x\right)+\sigma_{n, j}(x) z_{j} \mid x\right)=\frac{z_{j}}{v_{n, j}(x)}(1+o(1)),
$$

which shows that for all $j \in\{1, \ldots, J\}, t_{n, j} \rightarrow z_{j}$ as $n \rightarrow \infty$. Let us now turn to the random terms $W_{n, j}(x), j=1, \ldots, J$. Clearly,

$$
W_{n, j}(x)=\frac{1-a_{j} \gamma(x)}{\gamma(x)}\left(n h^{p} \alpha_{n}\right)^{1 / 2}\left(\frac{\hat{\varphi}_{a_{j}, n}^{\leftarrow}\left(\varphi_{a_{j}}\left(\varphi_{0}^{\leftarrow}\left(\alpha_{n} \mid x\right) \mid x\right)+\sigma_{n, j}(x) z_{j} \mid x\right)}{\varphi_{a_{j}}^{\leftarrow}\left(\varphi_{a_{j}}\left(\varphi_{0}^{\leftarrow}\left(\alpha_{n} \mid x\right) \mid x\right)+\sigma_{n, j}(x) z_{j} \mid x\right)}-1\right)(1+o(1))
$$

since, from (23),

$$
\frac{\varphi_{a_{j}}^{\leftarrow}\left(\varphi_{a_{j}}\left(\varphi_{0}^{\leftarrow}\left(\alpha_{n} \mid x\right) \mid x\right)+\sigma_{n, j}(x) z_{j} \mid x\right)}{\varphi_{0}^{\leftarrow}\left(\alpha_{n} \mid x\right)}=1+\frac{z_{j}}{\varphi_{0}^{\leftarrow}\left(\alpha_{n} \mid x\right) v_{n, j}(x)}(1+o(1))=1+o(1)
$$

Furthermore, we have

$$
W_{n, j}^{(0)}(x)=\frac{1-a_{j} \gamma(x)}{\gamma(x)}\left(n h^{p} \alpha_{n}\right)^{1 / 2}\left(\frac{\hat{\varphi}_{0, n}^{\leftarrow}\left(\alpha_{n} \mid x\right)}{\varphi_{0}^{\leftarrow}\left(\alpha_{n} \mid x\right)}-1\right) .
$$

As a consequence, applying Proposition 2 with $a_{J+1}=0, \alpha_{n, j}=\varphi_{a_{j}}\left(\varphi_{0}^{\overleftarrow{ }}\left(\alpha_{n} \mid x\right) \mid x\right)+\sigma_{n, j}(x) z_{j}$ for $j=1, \ldots, J$ and $\alpha_{n, J+1}=\alpha_{n}$ entails

$$
\left\{\left\{W_{n, j}(x)-W_{n, j}^{(0)}(x)\right\}_{j=1, \ldots, J}, W_{n, 0}^{(0)}(x)\right\}=M(x) \xi_{n}
$$


where $M$ is the $(J+1) \times(J+1)$ matrix defined by

$$
M(x)=\left(\begin{array}{c|c} 
& c_{1}(x) \\
\tilde{A}(x) & \vdots \\
& c_{J}(x) \\
\hline 0 \cdots 0 & 1
\end{array}\right)
$$

with

$$
\tilde{A}(x)=\operatorname{diag}\left(\frac{1-a_{1} \gamma(x)}{\gamma(x)}, \ldots, \frac{1-a_{J} \gamma(x)}{\gamma(x)}\right) \quad \text { and } \quad c_{j}=-\frac{1-a_{j} \gamma(x)}{\gamma(x)}, j \in\{1, \ldots, J\}
$$

and where $\xi_{n}$ is a $(J+1)$-random vector asymptotically Gaussian, centred with covariance $\|K\|_{2}^{2} \Sigma^{(2)}(x) / g(x)$. Since for each $j \in\{1, \ldots, J\}, t_{n, j} \rightarrow z_{j}$ as $n \rightarrow \infty$, the cumulative distribution function $\Phi_{n}$ converges to a centred Gaussian cumulative distribution function with covariance matrix $\|K\|_{2}^{2} M(x) \Sigma^{(2)}(x) M(x)^{t} / g(x)=\|K\|_{2}^{2} \gamma^{2}(x) \Sigma(x) / g(x)$, which is the desired result.

Proof of Corollary 2. Clearly, from Theorem 1 one has for $i=1,2$,

$$
\widehat{\operatorname{RCTM}}_{i, n}\left(\alpha_{n} \mid x\right)=\operatorname{RCTM}_{i}\left(\alpha_{n} \mid x\right)\left(1+\left(n h^{p} \alpha_{n}\right)^{-1 / 2} \xi_{i, n}\right)
$$

where the random vector $\left(\xi_{1, n}, \xi_{2, n}\right)$ is asymptotically Gaussian, centred with covariance matrix $\Sigma^{(3)}$ defined by

$$
\Sigma_{i, j}^{(3)}=i j \gamma^{2}(x) \frac{2-(i+j) \gamma(x)}{1-(i+j) \gamma(x)} \frac{\|K\|_{2}^{2}}{g(x)},(i, j) \in\{1,2\}^{2} .
$$

Hence,

$$
\begin{aligned}
\widehat{\operatorname{RCTV}}_{n}\left(\alpha_{n} \mid x\right)-\operatorname{RCTV}\left(\alpha_{n} \mid x\right) & =\left(n h^{p} \alpha_{n}\right)^{-1 / 2}\left(\operatorname{RCTM}_{2}\left(\alpha_{n} \mid x\right) \xi_{2, n}-2 \operatorname{RCTM}_{1}^{2}\left(\alpha_{n} \mid x\right) \xi_{1, n}\right. \\
& \left.-\left(n h^{p} \alpha_{n}\right)^{-1 / 2} \operatorname{RCTM}_{1}^{2}\left(\alpha_{n} \mid x\right) \xi_{1, n}^{2}\right), \\
& =\left(n h^{p} \alpha_{n}\right)^{-1 / 2}\left(\operatorname{RCTM}_{2}\left(\alpha_{n} \mid x\right) \xi_{2, n}\right. \\
& \left.-2 \operatorname{RCTM}_{1}^{2}\left(\alpha_{n} \mid x\right) \xi_{1, n}(1+o(1))\right) .
\end{aligned}
$$

Since for $a \in[0,1 /(2 \gamma(x)))$ and $b>0$ Lemma 1 entails that

$$
\operatorname{RCTM}_{a}^{b}\left(\alpha_{n} \mid x\right)=\frac{\left(\varphi_{0}^{\leftarrow}\left(\alpha_{n} \mid x\right)\right)^{a b}}{(1-a \gamma(x))^{b}}(1+o(1))
$$

one has

$$
\operatorname{RCTV}\left(\alpha_{n} \mid x\right)=\frac{\gamma^{2}(x)}{(1-2 \gamma(x))(1-\gamma(x))^{2}}\left(\varphi_{0}^{\leftarrow}\left(\alpha_{n} \mid x\right)\right)^{2}(1+o(1))
$$

Hence, from Theorem 1,

$$
\left(n h^{p} \alpha_{n}\right)^{1 / 2}\left(\frac{\widehat{\operatorname{RCTV}}_{n}\left(\alpha_{n} \mid x\right)}{\operatorname{RCTV}\left(\alpha_{n} \mid x\right)}-1\right)=\frac{4 \gamma(x)-2}{\gamma^{2}(x)} \xi_{1, n}(1+o(1))+\frac{(1-\gamma(x))^{2}}{\gamma^{2}(x)} \xi_{2, n}(1+o(1)),
$$

is asymptotically Gaussian, centred with variance

$$
\left(\frac{4 \gamma(x)-2}{\gamma^{2}(x)}, \frac{(1-\gamma(x))^{2}}{\gamma^{2}(x)}\right) \Sigma^{(3)}\left(\frac{4 \gamma(x)-2}{\gamma^{2}(x)}, \frac{(1-\gamma(x))^{2}}{\gamma^{2}(x)}\right)^{t}
$$

The conclusion follows. 
Proof of Corollary 3. Clearly, from Theorem 1 one has for $i=1,2,3$,

$$
\widehat{\operatorname{RCTM}}_{i, n}\left(\alpha_{n} \mid x\right)=\operatorname{RCTM}_{i}\left(\alpha_{n} \mid x\right)\left(1+\left(n h^{p} \alpha_{n}\right)^{1 / 2} \xi_{i, n}\right),
$$

where the random vector $\left(\xi_{1, n}, \xi_{2, n}, \xi_{3, n}\right)$ is asymptotically Gaussian, centred with covariance matrix $\Sigma^{(4)}$ defined by

$$
\Sigma_{i, j}^{(4)}=i j \gamma^{2}(x) \frac{\|K\|_{2}^{2}}{g(x)} \frac{2-(i+j) \gamma(x)}{1-(i+j) \gamma(x)},(i, j) \in\{1,2,3\}^{2} .
$$

From the proof of Corollary 2, it appears that

$$
\frac{\widehat{\operatorname{RCTV}}_{n}\left(\alpha_{n} \mid x\right)}{\operatorname{RCTV}\left(\alpha_{n} \mid x\right)}=1+\left(n h^{p} \alpha_{n}\right)^{-1 / 2}\left(\frac{4 \gamma(x)-2}{\gamma^{2}(x)} \xi_{1, n}(1+o(1))+\frac{(1-\gamma(x))^{2}}{\gamma^{2}(x)} \xi_{2, n}(1+o(1))\right),
$$

and thus

$$
\left(\frac{\widehat{\operatorname{RCTV}}_{n}\left(\alpha_{n} \mid x\right)}{\operatorname{RCTV}\left(\alpha_{n} \mid x\right)}\right)^{3 / 2}=1+\frac{3}{2}\left(n h^{p} \alpha_{n}\right)^{-1 / 2}\left(\frac{4 \gamma(x)-2}{\gamma^{2}(x)} \xi_{1, n}(1+o(1))+\frac{(1-\gamma(x))^{2}}{\gamma^{2}(x)} \xi_{2, n}(1+o(1))\right) .
$$

Clearly, from Theorem 1,

$\left(n h^{p} \alpha_{n}\right)^{1 / 2}\left(\frac{\widehat{\operatorname{RCTS}}_{n}\left(\alpha_{n} \mid x\right)}{\operatorname{RCTS}\left(\alpha_{n} \mid x\right)}-1\right)=\xi_{3, n}-\frac{3}{2}\left(\frac{4 \gamma(x)-2}{\gamma^{2}(x)} \xi_{1, n}(1+o(1))+\frac{(1-\gamma(x))^{2}}{\gamma^{2}(x)} \xi_{2, n}(1+o(1))\right)$,

is asymptotically Gaussian, centred with variance

$$
\left(-\frac{3}{2} \frac{4 \gamma(x)-2}{\gamma^{2}(x)},-\frac{3}{2} \frac{(1-\gamma(x))^{2}}{\gamma^{2}(x)}, 1\right) \Sigma^{(4)}\left(-\frac{3}{2} \frac{4 \gamma(x)-2}{\gamma^{2}(x)},-\frac{3}{2} \frac{(1-\gamma(x))^{2}}{\gamma^{2}(x)}, 1\right)^{t},
$$

and the result is proved.

Proof of Theorem 2. The proof is based on the following expansion:

$$
\frac{\sqrt{n h_{n}^{p} \alpha_{n}}}{\log \left(\alpha_{n} / \beta_{n}\right)}\left(\log \widehat{\operatorname{RCTM}}_{a, n}^{W}\left(\beta_{n} \mid x\right)-\log \operatorname{RCTM}_{a}\left(\beta_{n} \mid x\right)\right)=\frac{\sqrt{n h_{n}^{p} \alpha_{n}}}{\log \left(\alpha_{n} / \beta_{n}\right)}\left(Q_{n, 1}+Q_{n, 2}+Q_{n, 3}\right),
$$

with

$$
\begin{aligned}
Q_{n, 1} & =a \sqrt{n h_{n}^{p} \alpha_{n}}\left(\hat{\gamma}_{n}(x)-\gamma(x)\right), \\
Q_{n, 2} & =\frac{\sqrt{n h_{n}^{p} \alpha_{n}}}{\log \left(\alpha_{n} / \beta_{n}\right)} \log \left(\widehat{\operatorname{RCTM}}_{a, n}\left(\alpha_{n} \mid x\right) / \operatorname{RCTM}_{a}\left(\alpha_{n} \mid x\right)\right), \\
Q_{n, 3} & =\frac{\sqrt{n h_{n}^{p} \alpha_{n}}}{\log \left(\alpha_{n} / \beta_{n}\right)}\left(\log \operatorname{RCTM}_{a}\left(\alpha_{n} \mid x\right)-\log \operatorname{RCTM}_{a}\left(\beta_{n} \mid x\right)+a \gamma(x) \log \left(\alpha_{n} / \beta_{n}\right)\right) .
\end{aligned}
$$

Let us consider the three terms separately. Under the hypotheses of Theorem 2, it is clear that $Q_{n, 1} \stackrel{d}{\rightarrow} \mathcal{N}\left(0,(a v(x))^{2}\right)$. Theorem 1 implies that $\widehat{\operatorname{RCTM}}_{a, n}\left(\alpha_{n} \mid x\right) / \operatorname{RCTM}_{a}\left(\alpha_{n} \mid x\right) \stackrel{\mathbb{P}}{\rightarrow} 1$ when $n \rightarrow$ $\infty$ and thus

$$
Q_{n, 2}=\frac{\sqrt{n h_{n}^{p} \alpha_{n}}}{\log \left(\alpha_{n} / \beta_{n}\right)}\left(\frac{\widehat{\operatorname{RCTM}}_{a, n}\left(\alpha_{n} \mid x\right)}{\operatorname{RCTM}_{a}\left(\alpha_{n} \mid x\right)}-1\right)\left(1+o_{p}(1)\right)=\frac{O_{p}(1)}{\log \left(\alpha_{n} / \beta_{n}\right)} .
$$

As a consequence, $Q_{n, 2} \stackrel{\mathbb{P}}{\rightarrow} 0$ when $n \rightarrow \infty$. Finally, Lemma 2 entails $Q_{n, 3}=O\left(\sqrt{n h_{n}^{p} \alpha_{n}} \varepsilon_{a}\left(1 / \beta_{n} \mid x\right)\right)$ which converges to 0 by assumption. 

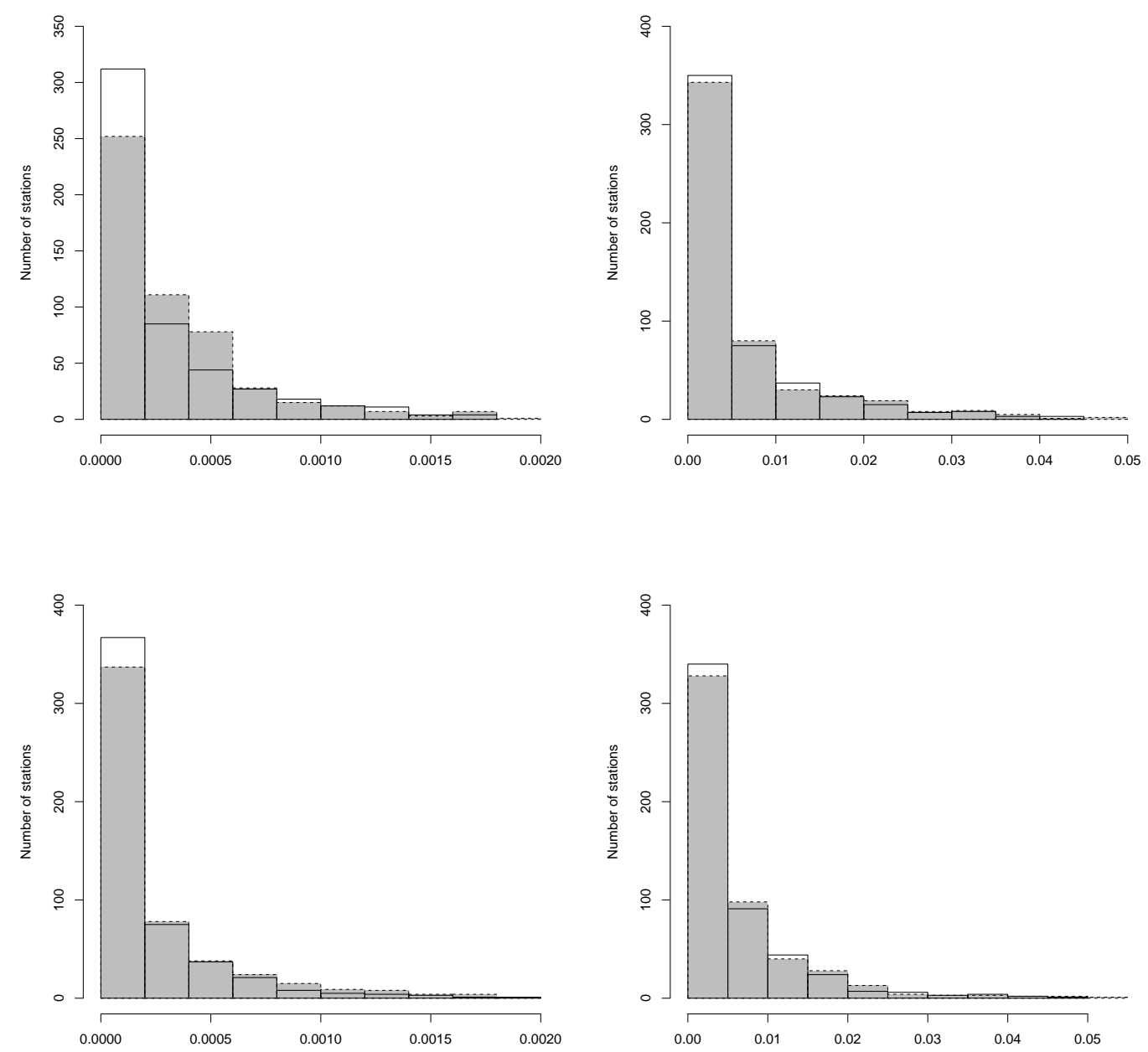

Figure 1: Histogram of the errors $V_{h_{o p t}, \alpha_{o p t}}\left(x_{t}\right)$ (solid line, white bars) and $V_{h_{e m p}, \alpha_{e m p}}\left(x_{t}\right)$ (dotted lines, grey bars) computed on simulated data (Burr distribution on the top panel, Fréchet distribution on the bottom panel). Upper left: $x_{e u c}$ and $\tau_{j}^{H a}$, upper right: $x_{a l t}$ and $\tau_{j}^{G}$, bottom left: $x_{\text {euc }}$ and $\tau_{j}^{G}$, bottom right: $x_{\text {alt }}$ and $\tau_{j}^{H a}$. 

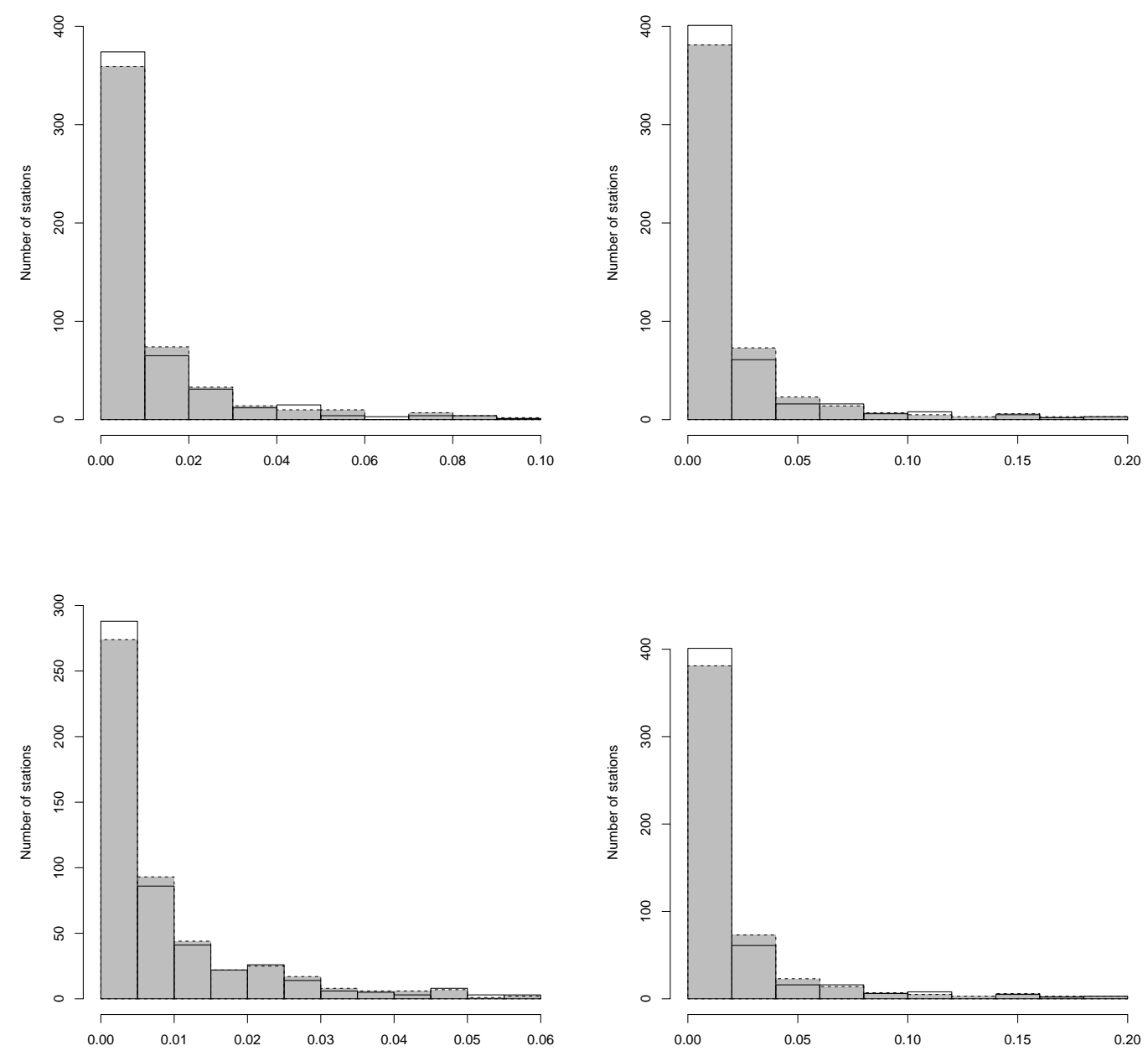

Figure 2: Histogram of the errors obtained for the extrapolated RCTE on simulated data. Set of parameters $\left(h_{\text {opt }}, \alpha_{\text {opt }}\right)$ : solid line and white bars, $\left(h_{e m p}, \alpha_{e m p}\right)$ : dotted lines and grey bars. Burr distribution: top panel, Fréchet distribution: bottom panel. Upper left: $x_{e u c}$ and $\tau_{j}^{H a}$, upper right: $x_{\text {alt }}$ and $\tau_{j}^{G}$, bottom left: $x_{e u c}$ and $\tau_{j}^{G}$, bottom right: $x_{\text {alt }}$ and $\tau_{j}^{H a}$. 

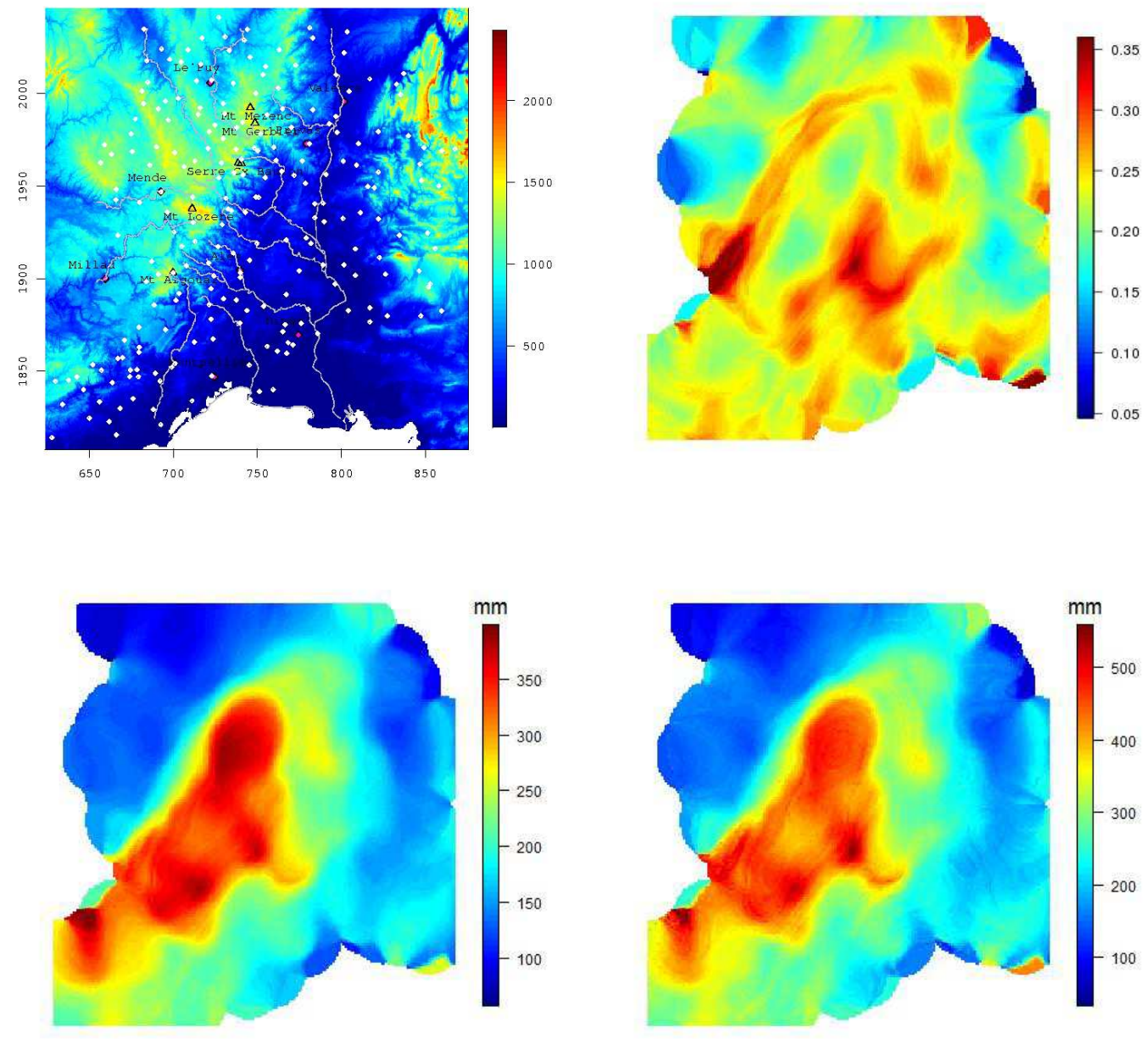

Figure 3: Upper left: map of the Cévennes-Vivarais region, horizontally: longitude (km), vertically: latitude $(\mathrm{km})$, the color scale represents the altitude $(\mathrm{m})$, the white dots represent some raingauge stations, upper right: $\hat{\gamma}_{n}(x)$, bottom left: $\widehat{\mathrm{RVaR}}_{n}^{W}\left(\beta_{n} \mid x\right)$ for a 100-year return period, bottom right: $\widehat{\mathrm{RCTE}}_{n}^{W}\left(\beta_{n} \mid x\right)$ for a 100-year return period. 